\title{
HEISENBERG MANIFOLDS AND THETA FUNCTIONS \\ BY
}

R. TOLIMIERI

\begin{abstract}
ABSTRACr. The algebraic structure of the $(2 n+1)$-dimensional Heisenberg group naturally induces a special class of differential operators whose solutions $(D f=0)$ are related to classical theta function theory.
\end{abstract}

1. Algebra. This section is purely algebraic. Its principal goal is to understand some of the algebraic concepts which lie at the heart of classical theta function theory. Firstly, we will recall the definition of the Heisenberg group and describe the structure of its automorphism group.

For an integer $n>0$, let $J_{2 n}$ be the $2 n \times 2 n$-matrix

$$
J_{2 n}=\left(\begin{array}{cc}
0 & E_{n} \\
-E_{n} & 0
\end{array}\right)
$$

where $E_{n}$ is the $n \times n$-identity matrix. Associated to $J_{2 n}$ is the nondegenerate, alternating, $R$-bilinear form on $R^{2 n}$ defined by

$$
J_{2 n}(\xi, \eta)=\eta^{t} \cdot J_{2 n} \cdot \xi, \quad \eta, \xi \in R^{2 n} .
$$

The Heisenberg group $N_{n}$ is defined as the $(2 n+1)$-dimensional Lie group whose underlying manifold is $R^{2 n} \times R$ and whose group multiplication is defined by the rule

$$
(\xi, z)(\eta, w)=\left(\xi+\eta, z+w+\frac{1}{2} J_{2 n}(\xi, \eta)\right)
$$

where $\xi, \eta \in R^{2 n}, z, w \in R$. In general, we denote a typical element $v \in N_{n}$ by $v=(\xi, z)$ with $\xi \in R^{2 n}, z \in R$. It is straightforward to verify that $N_{n}$ is a 2-step nilpotent, simply connected, analytic group having 1-dimensional center $Z=\{(0, z): z \in R\}$. The converse statement that every $(2 n+1)-$ dimensional, 2-step nilpotent, simply connected, analytic group having 1dimensional center is isomorphic to $N_{n}$ is deeper but also standard. Essentially, it is equivalent to the linear algebra result that for any real, alternating, nonsingular $2 n \times 2 n$-matrix $A$ we can write

$$
A=G^{t} J_{2 n} G, \quad G \in \mathrm{GL}(2 n, R) .
$$

For a proof, see Lang $[4$, p. 371].

Let $\mathbb{Q}_{n}$ denote the automorphism group of $N_{n}$. The 1-parameter subgroups of

Received by the editors May 5, 1976.

AMS (MOS) subject classifications (1970). Primary 22E25, 33A75; Secondary 58G99.

- American Mathematical Society 1978 
$N_{n}$ are all of the form $t \mapsto(t \xi, t z): R \rightarrow N_{n}$ for fixed $(\xi, z) \in N_{n}$ and hence are straight lines in the underlying manifold. It follows that every $\alpha \in \mathbb{Q}_{n}$ defines a linear transformation on this underlying manifold and hence can be described by a real $(2 n+1) \times(2 n+1)$-matrix. Moreover, since $Z$ is fixed by $\alpha$ we can write

$$
\alpha=\left(\begin{array}{cc}
S & 0 \\
\delta^{t} & d
\end{array}\right)
$$

where $S$ is a real $2 n \times 2 n$-matrix, $\delta^{t} \in R^{2 n}$ and $d \neq 0$ in $R$. Hence, $\alpha(\xi, z)=$ $\left(S \xi, d z+\delta^{t} \cdot \xi\right)$. The condition for $\alpha \in \mathbb{Q}_{n}$ is equivalent to the requirement

$$
J_{2 n}(S \xi, S \eta)=S^{t} J_{2 n} S(\xi, \eta)=d \cdot J_{2 n}(\xi, \eta),
$$

for all $\xi, \eta \in R^{2 n}$. In matrix language this becomes

$$
S^{t} J_{2 n} S=d J_{2 n} \text {. }
$$

Hence, $\mathbb{Q}_{n}$ consists of all matrices of the form given in (5a), satisfying (5c). Also, the identity component $\mathbb{Q}_{n}^{0}$ of $\mathbb{Q}_{n}$ consists of all $\alpha \in \mathbb{Q}_{n}$ with $d>0$.

We will now describe $\mathbb{Q}_{n}^{0}$ in greater detail. The symplectic group $\operatorname{Sp}(2 n, R)$ is defined by

$$
\mathrm{Sp}(2 n, R)=\left\{S \in \mathrm{GL}(2 n, R): S^{t} J_{2 n} S=J_{2 n}\right\} .
$$

The map $S \mapsto \alpha=\left(\begin{array}{l}S \\ 0\end{array}\right)$ identifies $\operatorname{Sp}(2 n, R)$ with a subgroup of $\mathbb{Q}_{n}^{0}$. We will assume this identification without further comment. Set

$$
\begin{aligned}
\mathscr{D}_{2 n} & =\left\{\left(\begin{array}{cc}
d E_{2 n} & 0 \\
0 & d^{2}
\end{array}\right): d>0\right\}, \\
\mathscr{R}_{2 n} & =\left\{\left(\begin{array}{cc}
d E_{2 n} & 0 \\
\delta^{t} & d^{2}
\end{array}\right): d>0, \delta \in R^{2 n}\right\}, \\
\operatorname{inn}\left(N_{n}\right) & =\left\{\left(\begin{array}{cc}
E_{2 n} & 0 \\
\delta^{t} & 1
\end{array}\right): \delta \in R^{2 n}\right\} .
\end{aligned}
$$

Clearly, the sets defined in (7) are all subgroups of $\mathbb{Q}_{n}^{0}$. $\mathfrak{R}_{2 n}$ can be characterized as the maximal, normal, solvable, analytic subgroup of $\mathbb{Q}_{n}^{0}$. Also, since $\Re_{2 n} \cap \operatorname{Sp}(2 n, R)=E_{2 n}$, we can form the semidirect product $\operatorname{Sp}(2 n, R) \ngtr$ $\Re_{2 n}$. It is almost immediate that

$$
\mathbb{Q}_{n}^{0}=\operatorname{Sp}(2 n, R) \ngtr \mathscr{R}_{2 n} .
$$

Denote by $\operatorname{inn}(\xi, z)$ the inner automorphism of $N_{n}$ given by

$$
\operatorname{inn}(\xi, z)((\eta, w))=(\xi, z)^{-1}(\eta, w)(\xi, z) .
$$

In matrix form we have 


$$
\operatorname{inn}(\xi, z)=\left(\begin{array}{cc}
E_{2 n} & 0 \\
J_{2 n}(\xi)^{t} & 1
\end{array}\right) .
$$

Hence, $\operatorname{inn}\left(N_{n}\right)$ denotes the group of all inner automorphisms of $N_{n}$. The following results are obvious.

$$
\mathscr{R}_{2 n}=\mathscr{D}_{2 n} \ngtr \operatorname{inn}\left(N_{n}\right), \quad \mathbb{Q}_{n}^{0}=\operatorname{Sp}(2 n, R) \ngtr \mathscr{D}_{2 n} \ngtr \operatorname{inn}\left(N_{n}\right) .
$$

Observe that the elements of $\operatorname{Sp}(2 n, R)$ and $\mathscr{D}_{2 n}$ commute with each other. Also set

$$
\operatorname{SL}\left(N_{n}\right)=\operatorname{Sp}(2 n, R) \ngtr \operatorname{inn}\left(N_{n}\right) .
$$

This is the subgroup of all $\alpha \in \mathbb{Q}_{n}^{0}$ whose restriction to $Z$ is the identity mapping.

We will now begin to investigate the first of two, essentially equivalent, algebraic concepts. In terms of each of these concepts, we can develop the 'analytic' results of the next section.

An element $\alpha \in \mathbb{Q}_{n}^{0}$ is called a CR-structure if $\alpha^{2} \equiv-E_{2 n} \bmod Z$. The set of all CR-structures is denoted by $\mathrm{CR}\left(N_{n}\right)$. $\mathbb{Q}_{n}^{0}$ acts on $\mathrm{CR}(N)$ by conjugation. For $\alpha \in \operatorname{CR}\left(N_{n}\right)$ and $\beta \in \mathbb{Q}_{n}^{0}$ we set $\beta[\alpha]=\beta^{-1} \alpha \beta$. Two CR-structures $\alpha_{1}$ and $\alpha_{2}$ are called conjugate whenever $\beta\left[\alpha_{1}\right]=\alpha_{2}$ for some $\beta \in \mathbb{Q}_{n}^{0}$. We denote the space of $\mathbb{Q}_{n}^{0}$-orbits of $\operatorname{CR}\left(N_{n}\right)$, or, equivalently, the space of conjugacy classes of CR-structures by $\mathrm{CR}\left(N_{n}\right) / \mathbb{Q}_{n}^{0}$, while we denote the conjugacy class containing an $\alpha \in \mathrm{CR}\left(N_{n}\right)$ by $\mathbb{Q}_{n}^{0}[\alpha]$. Observe that $\operatorname{CR}\left(N_{n}\right) \subset$ $\operatorname{SL}\left(N_{n}\right)$ and $\operatorname{SL}\left(N_{n}\right)[\alpha]=\mathbb{Q}_{n}^{0}[\alpha]$.

Eventually, we will describe $\operatorname{CR}\left(N_{n}\right)$ and $\operatorname{CR}\left(N_{n}\right) / \mathscr{Q}_{n}^{0}$ explicitly.

We begin with the following definition. Set

$$
\mathrm{CR}^{*}\left(N_{n}\right)=\mathrm{CR}\left(N_{n}\right) \cap \operatorname{Sp}(2 n, R) .
$$

We claim

$$
\begin{gathered}
\mathrm{CR}^{*}\left(N_{n}\right)=\left\{S \in \operatorname{Sp}(2 n, R): J_{2 n} S \text { is symmetric }\right\} . \\
\operatorname{CR}\left(N_{n}\right)=\operatorname{inn}\left(N_{n}\right)\left[\mathrm{CR}^{*}\left(N_{n}\right)\right] .
\end{gathered}
$$

To show (13) observe, $S^{t} J_{2 n} S=J_{2 n}$ and $S^{2}=-E_{2 n}$ imply $-S^{t} J_{2 n}=S^{t} J_{2 n}^{t}$ $=J_{2 n} S$. The converse follows by reversing these steps. To show (14) take $\alpha \in \operatorname{CR}\left(N_{n}\right)$ and write

$$
\alpha=\left(\begin{array}{cc}
S & 0 \\
\delta^{t} & 1
\end{array}\right)
$$

Let

$$
\beta=\left(\begin{array}{cc}
E_{2 n} & 0 \\
\gamma^{t} & 1
\end{array}\right) \in \operatorname{inn}\left(N_{n}\right) .
$$

To solve $\beta[S]=\alpha$ coincides with solving $\delta=\left(E_{2 n}-S\right) \gamma$. Since $S^{2}=$ 
$-E_{2 n}, E_{2 n}-S$ is nonsingular and $\gamma=\left(E_{2 n}-S\right)^{-1} \delta$. Hence, there is for each $\alpha \in \mathrm{CR}\left(N_{n}\right)$, a unique $S \in \mathrm{CR}^{*}\left(N_{n}\right)$ and a unique $\beta \in \operatorname{inn}\left(N_{n}\right)$, such that $\alpha=\beta[S]$ and (14) is verified.

The most important CR-structure for this paper is $J_{2 n}$. The following result describes the conjugacy class containing $J_{2 n}$.

LEMMA 1.1. For $\alpha \in \mathrm{CR}^{*}\left(N_{n}\right)$ to be conjugate to $J_{2 n}$ it is necessary and sufficient that $\alpha=\left(\begin{array}{cc}A & B \\ C & D\end{array}\right)$ where $B$ is symmetric and positive definite.

Proof. Firstly, if $\alpha$ is conjugate to $J_{2 n}$ then for some

$$
\beta=\left(\begin{array}{ll}
A_{1} & B_{1} \\
C_{1} & D_{1}
\end{array}\right) \in \mathrm{Sp}(2 n, R),
$$

$\beta\left[J_{2 n}\right]=\alpha$. It follows that $B=B_{1}^{\prime} B_{1}+D_{1}^{t} D_{1}$. Since $A_{1}^{t} D_{1}-C_{1}^{t} B_{1}=E_{n}$, there is no nonzero $\xi \in R^{n}$ satisfying $B_{1} \xi=D_{2} \xi=0$. Hence, $B$ is positive definite. Clearly, $B$ is symmetric as well and the first half of the lemma is verified. Conversely, if $\alpha=\left(\begin{array}{cc}A & B \\ C & D\end{array}\right)$ is in $\mathrm{CR}^{*}\left(N_{n}\right)$ with $B$ symmetric and positive definite, then $V B V^{t}=E_{n}$ for some $V \in \mathrm{GL}(n, R)$. The matrix

$$
S=\left(\begin{array}{cc}
V A & V B \\
-(V B)^{*} & 0
\end{array}\right) \in \operatorname{Sp}(2 n, R) \text { and } S[\alpha]=J_{2 n} .
$$

Define

$$
\mathcal{P}_{2 n}=\mathbb{Q}_{n}^{0}\left[J_{2 n}\right]
$$

The elements $\alpha \in \mathscr{P}_{2 n}$ will be called positive definite CR-structures.

We will now describe the space of conjugacy classes $\mathrm{CR}\left(N_{n}\right) / \mathbb{Q}_{n}^{0}$. Essentially, our main result refines the classical result that every $R$-quadratic form can be diagonalized. Let

$$
\delta_{n}^{+}=\text {the space of all nonsingular, symmetric, real, } n \times n \text {-matrices. }
$$

$\mathrm{GL}(n, R)$ acts on $\delta_{n}^{+}$by the rule

$$
X[F]=X^{t} F X, \quad F \in \mathcal{S}_{n}^{+}, \quad X \in \mathrm{GL}(n, R) .
$$

Two elements $F$ and $G$ in $\delta_{n}^{+}$are called similar, and we write $F \sim G$, whenever $X[F]=G$ for some $X \in \mathrm{GL}(n, R)$. Define

$$
\begin{aligned}
E_{p, q} & =\left(\begin{array}{cc}
E_{p} & 0 \\
0 & E_{q}
\end{array}\right), \quad p, q \geqslant 0 \text { in } \mathrm{Z} \text { and } p+q=n, \\
J_{2 n}(p, q) & =\left(\begin{array}{cc}
0 & E_{p, q} \\
-E_{p, q} & 0
\end{array}\right), \quad \mathscr{P}_{2 n}(p, q)=\left(\mathscr{Q}_{n}^{0}\right)\left[J_{2 n}(p, q)\right] .
\end{aligned}
$$

It is a well-known result of linear algebra (see Siegel $[8$, p. 85]) that every $F \in \delta_{n}^{+}$determines a unique pair of nonnegative integers $p, q$ satisfying $p+q=n$ and $F \sim E_{p, q}$. The pair $(p, q)$ is called the signature of $F$. We want 
to translate this result to CR-structures. Define

$$
\lambda G=\left(\begin{array}{cc}
0 & G \\
-G^{-1} & 0
\end{array}\right), \quad G \in \mathcal{S}_{n}^{+} .
$$

Clearly, $\lambda G \in \mathrm{CR}^{*}\left(N_{n}\right)$ and $J_{2 n}(p, q)=\lambda E_{p, q^{*}}$ Moreover, we have the following compatibility between the similarity relation on ${\delta_{n}^{+}}^{+}$and the conjugacy relation on $\operatorname{CR}\left(N_{n}\right)$.

LEMMA 1.2. For $F_{1}$ and $F_{2}$ in $S_{n}^{+}, F_{1}$ and $F_{2}$ are similar if and only if $\lambda F_{1}$ and $\lambda F_{2}$ are conjugate as $\mathrm{CR}$-structures.

Proof. Recall $X^{*}=\left(x^{t}\right)^{-1}, X \in \mathrm{GL}(n, R)$. Set

$$
X^{\prime}=\left(\begin{array}{cc}
X & 0 \\
0 & X^{*}
\end{array}\right), \quad X \in \mathrm{GL}(n, R) .
$$

Clearly, $X^{\prime} \in \operatorname{Sp}(2 n, R)$. Suppose $F_{1} \sim F_{2}$. Then $F_{1}=X F_{2} X^{t}$ for some $X \in$ $\mathrm{GL}(n, R)$. But this implies $X^{\prime}\left[\lambda F_{1}\right]=\lambda F_{2}$. Conversely, if $\lambda F_{1} \sim \lambda F_{2}$ as CRstructures then $\beta\left[\lambda F_{2}\right]=\lambda F_{1}$ for some $\beta=\left({ }_{C}^{A}{ }_{D}^{B}\right) \in \mathrm{Sp}(2 n, R)$. Hence,

$$
F_{2}=B F_{1}^{-1} B^{t}+A F_{1} A^{t}=\left(A+i B F^{-1}\right) F\left(A^{t}-i F^{-1} B^{t}\right) \text {. }
$$

It follows that $F_{1}$ and $F_{2}$ are Hermitian similar, and since they are real matrices they are similar.

(For the definition of Hermitian similar and the result stated in the proof, see Lang [4, p. 342].)

From the lemma we have that the orbits $\mathscr{P}_{2 n}(p, q)$ are disjoint and each $\lambda F, F \in \mathcal{S}_{n}^{+}$, is contained in the union of these orbits. Our next theorem tells us that the union of these orbits is all of $\operatorname{CR}\left(N_{n}\right)$.

TheOREM 1.3. $\mathrm{CR}\left(N_{n}\right) / \mathbb{Q}_{n}^{0}=\left\{\mathscr{P}_{2 n}(p, q): p, q \geqslant 0\right.$ in $\mathrm{Z}$ and $\left.p+q=n\right\}$.

Proof. It suffices to show that each

$$
S=\left(\begin{array}{cc}
A & B \\
C & -A^{t}
\end{array}\right) \in \mathrm{CR}^{*}\left(N_{n}\right)
$$

(as in (13)) is conjugate to some $\lambda F, F \in \delta_{n}^{+}$. For $B$ nonsingular we have

$$
\beta[S]=\lambda B \quad \text { where } \beta=\left(\begin{array}{cc}
-A & -B \\
B^{-1} & 0
\end{array}\right) \in \operatorname{Sp}(2 n, R) .
$$

The general case will be reduced to the case above. Since $S$ generates a compact subgroup of $\mathrm{Sp}(2 n, R)$, by a classical result $S$ is conjugate in $\operatorname{Sp}(2 n, R)$ to an element in $\operatorname{Sp}(2 n, R) \cap \mathcal{O}(2 n, R)$. For an explicit proof see Igusa [2, p. 23]. Hence, we can assume $S$ is of the form $S=\left({ }_{-B}^{A} A_{A}^{B}\right)$ where $B$ is symmetric, $A$ is alternating, $A^{2}-B^{2}=-E_{n}$ and $A B+B A=0$. We want to find a

$$
T=\left(\begin{array}{cc}
E_{n} & G \\
0 & E_{n}
\end{array}\right)
$$


with $G$ symmetric satisfying

$$
T[S]=\left(\begin{array}{ll}
A_{1} & B_{1} \\
C_{1} & D_{1}
\end{array}\right)
$$

with $B_{1}$ nonsingular. This is equivalent to solving

$$
B_{1}=-A G+G B G+B+G A
$$

with $G$ symmetric and $B_{1}$ nonsingular. Since $B$ is symmetric it can be diagonalized by some orthogonal matrix $O$, i.e., $O^{\prime} B O$ is diagonal. Condition (a) is independent of such a change, hence we can assume $B$ is diagonal to begin with. Write

$$
B=\left(\begin{array}{c|c}
\delta(b) & 0 \\
\hline 0 & 0
\end{array}\right)
$$

where

$$
\delta(b)=\left[\begin{array}{lll}
b_{1} & & 0 \\
0 & \cdot & b_{l}
\end{array}\right)
$$

is a diagonal $l \times l$ matrix and $b_{j} \neq 0,1 \leqslant j \leqslant l$. From $A^{2}-B^{2}=-E_{n}$ it follows that

$$
A=\left(\begin{array}{cc}
A_{1} & 0 \\
0 & A_{4}
\end{array}\right)
$$

where $A_{1}$ is an $l \times l$-matrix, $A_{4}^{\prime}=-A_{4}$ and $A_{4}^{2}=-E_{n-l}$. It follows that $A_{4}$ is orthogonal and is conjugate by an orthogonal matrix to a matrix consisting of the $2 \times 2$ blocks $J_{2}$ down the diagonal. A symmetric matrix $S_{4} \in \delta_{n-1}$ can then be found satisfying $-A_{4} S_{4}+S_{4} A_{4}$ is nonsingular. Taking

$$
G=\left(\begin{array}{cc}
0 & 0 \\
0 & S_{4}
\end{array}\right)
$$

satisfies condition (a).

We will now consider the automorphism group of a CR-structure $\alpha$. For $\alpha \in \operatorname{CR}\left(N_{n}\right)$, put

$$
\operatorname{aut}(\alpha)=\left\{\beta \in \mathbb{Q}_{n}^{0}: \beta^{-1} \alpha \beta=\alpha\right\} .
$$

The elements of aut $(\alpha)$ will be called $\alpha$-automorphisms. If $\beta\left[\alpha_{1}\right]=\alpha_{2}$ for $\alpha_{j} \in \mathrm{CR}\left[N_{n}\right]$ and $\beta \in \mathbb{Q}_{n}^{0}$, then clearly $\beta^{-1}$ aut $\left(\alpha_{1}\right) \beta=\operatorname{aut}\left(\alpha_{2}\right)$. Hence, to compute the automorphism groups of CR-structures it suffices to compute the automorphism groups of the CR-structures $J_{2 n}(p, q)$.

We begin by considering aut $\left(J_{2 n}\right)$. Firstly, set

$$
K=\operatorname{Sp}(2 n, R) \cap \vartheta(2 n, R) .
$$


Obviously, for $S \in \operatorname{Sp}(2 n, R)$, we have $S \in K$ if and only if $S^{t} J_{2 n}=J_{2 n} S^{t}$, which implies

$$
K=\left\{\left(\begin{array}{cc}
A & B \\
-B & A
\end{array}\right) \in \operatorname{Sp}(2 n, R)\right\}
$$

As we have mentioned in the proof of Theorem 1.3, $K$ has been previously studied. It is not hard to show that $K$ is a maximal compact subgroup of $\operatorname{Sp}(2 n, R)$ and any maximal compact subgroup of $\operatorname{Sp}(2 n, R)$ is conjugate in $\mathrm{Sp}(2 n, R)$ to $K$. The next lemma is almost obvious.

LEMMA 1.4. $\operatorname{aut}\left(J_{2 n}\right)=K \ngtr \mathscr{D}_{2 n}$.

Proof. For

$$
\alpha=\left(\begin{array}{cc}
S & 0 \\
\delta^{t} & d
\end{array}\right) \in \operatorname{aut}\left(J_{2 n}\right)
$$

we have

$$
S^{-1} J_{2 n} S=S^{t} J_{2 n} S=J_{2 n} \quad \text { and } \quad \delta^{t}=\delta^{t} S^{-1} J_{2 n} S .
$$

Hence, $\delta=0$ and

$$
\alpha=\left(\begin{array}{ccc}
1 / \sqrt{d} & S & 0 \\
0 & & 1
\end{array}\right)\left(\begin{array}{cc}
\sqrt{d} E_{2 n} & 0 \\
0 & d
\end{array}\right)
$$

This proves the lemma.

In the same way we can verify the next lemma.

LEMMA 1.5. $\operatorname{aut}\left(J_{2 n}(p, q)\right)=K_{p, q} \ngtr \mathscr{D}_{2 n}$ where

$$
K_{p, q}=\left\{\left(\begin{array}{cc}
A & B \\
-E_{p, q} B E_{p, q} & E_{p, q} A E_{p, q}
\end{array}\right) \in \operatorname{Sp}(2 n, R)\right\} .
$$

Define

$$
\operatorname{aut}(p, q)=\operatorname{aut}\left(J_{2 n}(p, q)\right), \quad G(p, q)=\operatorname{aut}(p, q) \ngtr N_{n},
$$

and, in general, if $\alpha \in \operatorname{CR}\left(N_{n}\right)$,

$$
G(\alpha)=\operatorname{aut}(\alpha) \ngtr N_{n} .
$$

For congruent CR-structures $\alpha_{1}$ and $\alpha_{2}=\beta\left[\alpha_{1}\right]$ the mapping

$$
(\gamma, v) \mapsto\left(\beta^{-1} \gamma \beta, \beta^{-1}(v)\right)
$$

defines an isomorphism from $G\left(\alpha_{1}\right)$ onto $G\left(\alpha_{2}\right)$. We will now consider the converse. Our main tools come from Jacobson [3], primarily Theorem 10, p. 81 and the theorem of Malcev and Harish-Chandra, p. 92 . Corollary 2 on p. 72 is used as well. These results will be assumed without further comment.

The case $n=2$ is trivial, hence we assume until the end of the next theorem that $n \geqslant 3$. In this case both the orthogonal group $\Theta(p, q)$ of $E_{p, q}$ and its 
commutator subgroup $\Omega(p, q)$ are irreducible (see O'Meara [7, p. 111]). The map

$$
A \mapsto A^{\prime}=\left(\begin{array}{cc}
A & 0 \\
0 & E_{p, q} A E_{p, q}
\end{array}\right)
$$

defines an isomorphism of $\Theta(p, q)$ into aut $(p, q)$. Since $J_{2 n} \in \operatorname{aut}(p, q)$ it follows that aut $(p, q)$ is irreducible. Thus $N_{n}$ is the nil-radical of $G(p, q)$. Also, $\operatorname{aut}(p, q)=[\operatorname{aut}(p, q), \operatorname{aut}(p, q)] \ngtr z_{p, q}$, where $z_{p, q}$ is the center of $\operatorname{aut}(p, q)$.

THeOREM 1.6. Two CR-structures $\alpha_{1}$ and $\alpha_{2}$ are conjugate, up to multiplication by $-E_{2 n}$, if and only if the corresponding groups $G\left(\alpha_{1}\right)$ and $G\left(\alpha_{2}\right)$ are Lie group isomorphic.

Proof. Without loss of generality we can assume $\alpha_{1}=J_{2 n}(p, q)$ and $\alpha_{2}=J_{2 n}(r, s)$. Assume $\rho: G(p, q) \rightarrow G(r, s)$ is a Lie group isomorphism. Firstly, $\rho\left(N_{n}\right)=N_{n}$ and, by our discussion above, $\rho([\operatorname{aut}(p, q)$, aut $(p, q)])$ is a Levi-factor of $G(r, s)$. The conjugacy theory of Levi-factors implies we can assume without loss of generality that

$$
\rho([\operatorname{aut}(p, q), \operatorname{aut}(p, q)])=[\operatorname{aut}(r, s), \operatorname{aut}(r, s)] .
$$

Thus, for some $S \in \mathrm{Sp}(2 n, R), S^{-1} \operatorname{aut}(p, q) S \supset[\operatorname{aut}(r, s)$, aut $(r, s)]$ and, hence, $\Omega^{\prime}(r, s)$ centralizes $S^{-1} J_{2 n}(p, q) S$. As a result, $\Omega(r, s) \subset \theta(F)$, where $F=X E_{p, q} \bar{X}^{t}$, where $X=A^{t}-i C^{t} E_{p, q}$. Since both $F$ and $E_{p, q}$ are real matrices it follows that $F$ and $E_{p, q}$ are similar. Hence, $\Omega(r, s)$ is contained in a conjugate of $\Theta(p, q)$ in $\mathrm{GL}(n, R)$. Thus, $\Omega(G) \subset \mathcal{O}(p, q)$ where $G$ is similar to $E_{r, s}$. By Schur's lemma, since $\Omega(G)$ is irreducible and commutes with $E_{p, q} G$, we have $G=\varepsilon \cdot E_{p, q}, \varepsilon \neq 0$ in $R$, and the theorem follows.

Let $\mathrm{C}^{n}$ denote the $n$-fold cartesian product of the complex numbers $C$. In all that follows we shall fix the identifications

$$
R^{2 n} \simeq R^{n} \oplus R^{n} \simeq \mathrm{C}^{n},
$$

defined by setting $\left(\xi_{1}, \xi_{2}\right)=\xi_{1}+i \xi_{2}, \xi_{1}, \xi_{2} \in R^{n}$. Thus $J_{2 n}(\xi, \eta)=\operatorname{im} \xi \bar{\eta}$, $\xi, \eta \in R^{n}$. It is obvious, but important, to note that the symplectic group $\operatorname{Sp}(2 n, R)$ does not act as a group of analytic automorphisms of $\mathrm{C}^{n}$ (assuming the above identification). A simple computation shows that $S \in \operatorname{Sp}(2 n, R)$ is an analytic automorphism of $C^{n}$ if and only if it satisfies the property $S^{+} J_{2 n}=J_{2 n} S^{+}$. Hence the group $K$ defined above [20] describes all the elements of $\operatorname{Sp}(2 n, R)$ which are analytic automorphisms.

We will now describe the lattices of $N_{n}$. A lattice of $N_{n}$ will be defined as a discrete subgroup $\Gamma$ of $N_{n}$ such that the space of right cosets $\Gamma \backslash N_{n}=\left\{\Gamma_{v}\right.$ : $\left.v \in N_{n}\right\}$ is compact and $[\Gamma, \Gamma]=\Gamma \cap Z$. This last condition is not usual but 
permits a simpler exposition. Furthermore, the lattices of $N_{n}$ can be characterized as the collection of all nonabelian subgroups of $N_{n}$ on $2 n$-generators. The main tool in structuring the lattices of $N_{n}$ is $\mathbb{Q}_{n}^{0}$. Set

$$
\mathcal{L}=\text { collection of all lattices of } N_{n}
$$

and define the action of $\mathbb{Q}_{n}^{0}$ on $\mathcal{E}$ by

$$
\Gamma^{\alpha}=\alpha(\Gamma), \quad \alpha \in \mathbb{Q}_{n}^{0} \text { and } \Gamma \in \mathcal{L} .
$$

Since $\Gamma \cap Z$ is a nontrivial discrete subgroup of $Z$ whenever $\Gamma \in \mathcal{L}$, we can find a uniquely determined positive real number $\beta(\Gamma)$ defined by the condition

$$
\Gamma \cap Z=\{(0, \beta(\Gamma) \cdot m): m \in Z\}
$$

The description of $\mathcal{L}$ requires, as we shall see below, familiarity with those lattices $D$ of $C^{n}$ which admit Riemann forms. We will say more about this in the next section. For now we shall consider just what we need for describing L.

A lattice of $C^{n}$ is a discrete subgroup $D$ of $C^{n}$ where $D \backslash C^{n}$ is compact. The lattices in $\mathcal{L}$ are a source of lattices in $C^{n}$ of a very special kind. Consider the short exact sequence

$$
1 \rightarrow Z \rightarrow N_{n} \stackrel{\pi}{\rightarrow} \mathrm{C}^{n} \rightarrow 1
$$

where $\pi(\xi, z)=\xi$. Recall we are identifying $R^{2 n}$ with $C^{n}$. By Malcev [6] whenever $\Gamma \in \mathcal{L}$ we have that $\pi(\Gamma)$ is a lattice of $\mathrm{C}^{n}$. Let $S \in \operatorname{Sp}(2 n, R)$ and $\Gamma \in \mathcal{L}$. Then $S(\Gamma) \in \mathcal{L}$ and $S$ induces a diffeomorphism between $\Gamma \backslash N_{n}$ and $S(\Gamma) \backslash N_{n}$. However, in general, as noted above, $S$ is not an analytic isomorphism of $\mathrm{C}^{n}$ and, hence, as complex manifolds $\pi(\Gamma) \backslash C^{n}$ and $S(\pi(\Gamma)) \backslash$ $\mathrm{C}^{n}$ are not necessarily analytically isomorphic.

The condition $[\Gamma, \Gamma]=\Gamma \cap Z$ (in fact, simply the fact that $[\Gamma, \Gamma]$ is a nontrivial discrete subgroup of $Z$ ) places further conditions on $\pi(\Gamma)$. For $v=(\xi, z), w=(\eta, w) \in N_{n},[v, w]=\left(0, J_{2 n}(\xi, \eta)\right)$. Hence, for any $\xi, \eta \in$ $\pi(\Gamma)$ we have

$$
J_{2 n}(\xi, \eta) \in \beta(\Gamma) \cdot Z
$$

In fact we will show in Lemma 1.7 below that

$$
J_{2 n}(\pi(\Gamma), \pi(\Gamma))=\beta(\Gamma) \cdot \mathrm{Z} .
$$

With this in mind, we call a lattice $D$ of $C^{n}$ a Heisenberg lattice whenever $J_{2 n}(D, D)=l \cdot \mathrm{Z}$ for some $l>0$ in $R$. Denote the collection of all Heisenberg lattices of $\mathrm{C}^{n}$ by $H \varrho\left(\mathrm{C}^{n}\right)$.

For elements $\xi_{1}, \xi_{m} \in \mathrm{C}^{n}$ denote the Z-module of $\mathrm{C}^{n}$ spanned by these elements by $\left[\xi_{1}, \ldots, \xi_{m}\right]$. 
LEMMA 1.7. For a lattice $D$ of $\mathrm{C}^{n}$ satisfying $J_{2 n}(D, D) \subset l \cdot \mathrm{Z}, l>0$, we have $D \in H \mathcal{L}\left(\mathrm{C}^{n}\right)$.

Proof. Dividing the elements of $D$ by $\sqrt{l}$ we see that there is no loss of generality in assuming $l=1$. Let $l_{1}$ be the smallest positive integer in $J_{2 n}(D, D)$ and $l_{1}=J_{2 n}\left(\xi_{1}, \eta_{1}\right)$ for $\xi_{1}, \eta_{1} \in D$. Set

$$
\left[\xi_{1}, \eta_{1}\right]^{\perp}=\left\{\xi \in D: J_{2 n}\left(\xi, \xi_{1}\right)=J_{2 n}\left(\eta_{1}, \xi\right)=0\right\} \text {. }
$$

We claim $D=\left[\xi_{1}, \eta_{1}\right] \oplus\left[\xi_{1}, \eta_{1}\right]^{\perp}$. For $\xi \in D$, the Euclidean algorithm implies

$$
0 \leqslant m l_{1}+J_{2 n}\left(\xi_{1}, \xi\right)=r<l_{1}, \quad m, r \in Z .
$$

Hence, $0 \leqslant J_{2 n}\left(\xi_{1}, \xi+m \eta_{1}\right)=r<l_{1}$, which contradicts the definition of $l_{1}$ unless $r=0$. Hence, $\xi+m \eta_{1} \in\left[\xi_{1}\right]^{\perp}$. In the same way, $\xi+m^{\prime} \xi_{1} \in\left[\eta_{1}\right]^{\perp}$ for some $m^{\prime} \in Z$. Thus $\xi+m^{\prime} \xi_{1}+m \eta_{1} \in\left[\xi_{1}, \eta_{1}\right]^{\perp}$ and our claim is verified. We are done once we show that for $\xi, \eta \in\left[\xi_{1}, \eta_{1}\right]^{\perp}, l_{1} / J_{2 n}(\xi, \eta)$. Again by the Euclidean algorithm

$$
0 \leqslant m l_{1}+J_{2 n}(\xi, \eta)=r<l_{1}, \quad m, r \in Z,
$$

and, as before, $r=0$ and $l_{1} / J_{2 n}(\xi, \eta)$.

The proof of the previous lemma is the main step in an induction proof (see Lang [5, p. 64]) of the next result.

LEMMA 1.8. For $D \in H \mathcal{L}\left(C^{n}\right)$ we can write $D$ as the $J_{2 n}$-orthogonal direct sum $D=\left[\xi_{1}, \eta_{1}\right] \oplus \cdots \oplus\left[\xi_{n}, \eta_{n}\right]$ of 2-dimensional Z-modules $\left[\xi_{j}, \eta_{j}\right]$. Let $l_{j}=$ $J_{2 n}\left(\xi_{j}, \eta_{j}\right)$; then the decomposition can be taken so that $l_{j} \in R_{+}^{x}$ and $l_{j} / l_{j+1}$, i.e., $l_{j+1} / l_{j} \in \mathbf{Z}$.

Moreover, these numbers $l_{1}, \ldots, l_{n}$ are uniquely determined by $D$.

Corollary. $\pi \mathcal{L}=H \mathcal{L}\left(\mathrm{C}^{n}\right)$.

Proof. By Lemma 1.7 and the discussion preceding it, whenever $\Gamma \in \mathcal{L}$, $\pi \Gamma \in H \mathcal{L}\left(\mathrm{C}^{n}\right)$. Conversely, let $D \in H \mathcal{L}\left(\mathrm{C}^{n}\right)$ and $D=\left[\xi_{1}, \eta_{1}\right] \oplus \cdots \oplus$ $\left[\xi_{n}, \eta_{n}\right]$ as in Lemma 1.8. In $N_{n}$ choose preimages of $\xi_{j}, \eta_{j}$ and set $\Gamma=$ the group generated by these preimages. Then $\Gamma \in \mathcal{L}$ and $\pi \Gamma=D$.

Corollary. For $\Gamma \in \mathcal{L}, \beta(\Gamma) Z=J_{2 n}(\pi \Gamma, \pi \Gamma)$.

Set

$$
\mathrm{Z}_{n}^{\#}=\left\{l=\left(1, l_{2}, \ldots, l_{n}\right) \in \mathrm{Z}_{+}^{x}: l_{j} / l_{j+1}\right\}
$$

Let $e_{j}, 1 \leqslant j \leqslant 2 n$, denote the $2 n$-tuple consisting of all zeros except for a one in the $j$ th place. For $l \in Z_{n}^{\#}$ define

$$
D(l)=\left[e_{1}, \ldots, e_{n}, e_{n+1}, l_{2} e_{n+2}, \ldots, l_{n} e_{2 n}\right] .
$$

Clearly, $D(l) \in H \mathcal{L}\left(\mathrm{C}^{n}\right)$ and $J_{2 n}(D(l), D(l))=\mathrm{Z}$. 
THEOREM 1.9. For each $D \in H \mathcal{L}\left(C^{n}\right)$ there exist a unique $l \in Z_{n}^{\#}$, a unique $d \neq 0$ in $R_{+}$and an $\alpha \in \mathrm{Sp}(2 n, R)$ such that $D=\alpha(d \cdot D(l))$.

Proof. We can assume $J_{2 n}(D, D)=Z$. Then $D=\left[\xi_{1}, \eta_{1}\right] \oplus \cdots \oplus\left[\xi_{n}, \eta_{n}\right]$ as in Lemma 1.8, and if $l_{j}=\left[\xi_{j}, \eta_{j}\right]$ we have $l \in Z_{n}^{\#}$. Set $\xi=\left(\xi_{1}, \ldots, \xi_{n}\right)$ and $\eta^{\prime}=\left(\eta_{1}, \eta_{2} / l_{2}, \ldots, \eta_{n} / l_{n}\right)$. Regarding $\xi, \eta^{\prime} \in R^{2 n}$, it is easy to see that the matrix $\alpha=\left(\xi \eta^{\prime}\right) \in \operatorname{Sp}(2 n, R)$ and $D=\alpha(D(l))$. The uniqueness is also contained in Lemma 1.8 .

Set $e_{j}^{\prime}=\left(e_{j}, 0\right) \in N_{n}$. For $l \in Z_{n}^{\#}$ define

$$
\Gamma(l)=\operatorname{gp}\left\{e_{1}^{\prime}, \ldots, e_{n}^{\prime}, e_{n+1}^{\prime}, l_{2} e_{n+2}^{\prime}, \ldots, l_{n} e_{2 n}^{\prime}\right\}
$$

In particular, set $\Gamma_{n}=\Gamma(1,1, \ldots, 1)$. For $d \in R_{+}^{x}$ we put

$$
d v=\left(\begin{array}{cc}
d E_{2 n} & 0 \\
0 & d^{2}
\end{array}\right) v, \quad v \in N_{n} .
$$

THEOREM 1.10. For $\Gamma \in \mathcal{L}$, there exist a unique $l \in Z_{n}^{\#}$, a unique $d \in R_{+}^{x}$ and an $\alpha \in \operatorname{SL}\left(N_{n}\right)$ such that $\Gamma=\alpha(d \cdot \Gamma(l))$.

Proof. Without loss of generality we can assume $\beta(\Gamma)=1$. Then $D=$ $\pi(\Gamma) \in H \mathcal{L}\left(C^{n}\right)$ and $J_{2 n}(D, D)=Z$. Hence, $D=\alpha(D(l))$, where $\alpha \in$ $\operatorname{Sp}(2 n, R)$ and $l \in Z_{n}^{\#}$. Since $\alpha \in \operatorname{aut}\left(N_{n}\right)$ and commutes with $\pi$, we can just as well assume $D=D(l)$. There exists $\delta=\left(\delta_{1}, \ldots, \delta_{2 n}\right) \in R^{2 n}$ so that

$$
\Gamma=\operatorname{gp}\left\{\left(e_{j}, \delta_{j}\right),\left(l_{j} e_{n+j}, l_{j} \delta_{n+j}\right)\right\}_{j=1, \ldots, n} .
$$

Hence, $\Gamma=\operatorname{inn}\left(J_{2 n}(\delta), 0\right) \times(\Gamma(l))$, which proves the theorem.

Since

$$
\left(\begin{array}{cc}
d E_{2 n} & 0 \\
0 & d^{2}
\end{array}\right) \in \mathbb{Q}_{n}^{0}
$$

it follows that the groups $\Gamma(l), l \in Z_{n}^{\#}$, determine $\mathcal{L}$ up to $\mathbb{Q}_{n}^{0}$.

Let $\Gamma \in \mathcal{L}$. In the next section we shall consider various function spaces on $N_{n}$ periodic with respect to left multiplication by elements from $\Gamma$. The remainder of this chapter is spent developing some of the algebraic machinery which will be applied to analyzing the structure of these function spaces. For $D \in H\left(C^{n}\right)$ write $D=\alpha(d \cdot D(l))$ as in Theorem 1.9. The $l \in Z_{n}^{\#}$ and $d>0$ are uniquely determined by $D$. Define

$$
\mathscr{P}(D)=\left(1 \cdot l_{2} \cdots l_{n}\right)^{2} .
$$

We call $\mathcal{P}(D)$ the Pfaffian of $D$. Also, we put

$$
\beta(D)=d^{2} \text {. }
$$

$\beta(D)$ is the unique positive number satisfying

$$
J_{2 n} \beta(D, D)=\beta(D) \cdot Z \text {. }
$$


Analogously, for $\Gamma \in \mathcal{L}$ we can write $\Gamma=\alpha(d \cdot \Gamma(l))$ as in Theorem 1.14. The Pfaffian $\mathcal{P}(\Gamma)$ of $\Gamma$ is defined by setting

$$
\mathscr{P}(\Gamma)=\left(1 \cdot l_{2} \cdots l_{n}\right)^{2} \text {. }
$$

Clearly, for $\Gamma \in \mathcal{L}, \mathscr{P}(\Gamma)=\mathscr{P}(\pi \Gamma)$. Moreover, we have previously defined $\beta(\Gamma)>0$ by the condition

$$
\Gamma \cap Z=\beta(\Gamma) \cdot Z Z
$$

where $Z Z=\{(0, m): m \in Z\}$. Clearly,

$$
\beta(\Gamma)=d^{2}=\beta(\pi(\Gamma))
$$

A duality theory for $H \mathcal{L}\left(\mathrm{C}^{n}\right)$, and correspondingly for $\mathcal{L}$, will now be developed. For $D \in H \mathcal{L}\left(\mathrm{C}^{n}\right)$ put

$$
\hat{D}=\left\{\xi \in C^{n}: J_{2 n}(\xi, \eta) \in \beta(D) \cdot Z \text { for all } \eta \in D\right\} .
$$

We call $\hat{D}$ the dual of $D$.

LEMMA 1.11. For $D \in H \mathcal{L}\left(C^{n}\right), \hat{D} \in H \mathcal{L}\left(\mathrm{C}^{n}\right), D \subset \hat{D}$ and $O(\hat{D} / D)=$ $\mathscr{P}(D)$.

Proof. Let $D=\alpha(d \cdot D(l))$ as above. Then $\hat{D}=\alpha(d \cdot \hat{D}(l))$. Hence the lemma is proved once we show its validity for $D(l)$. In this case,

$$
\hat{D}=\operatorname{gp}\left\{e_{1} e_{2} / l_{2}, \ldots, e_{n} / l_{n}, e_{n+1}, \ldots, e_{2 n}\right\} \text {. }
$$

Then $J_{2 n}(\hat{D}, \hat{D})=1 / l_{n} \cdot \mathrm{Z}$ and, hence, $\hat{D} \in H \mathcal{L}\left(\mathrm{C}^{n}\right)$. Clearly, $D \subset \hat{D}$ and $O(\hat{D} / D)=\left(1 \cdot l_{2} \cdots l_{n}\right)^{2}=\mathscr{P}(D)^{2}$.

A relative duality theory will also be required. For $D \subset D^{\prime}$, both lattices in $H \mathcal{L}\left(\mathrm{C}^{n}\right)$, put

$$
\widehat{D^{\prime} \mid D}=\left\{\xi \in \mathrm{C}^{n}: J_{2 n}(\xi, \eta) \in \beta\left(D^{\prime}\right) \mathrm{Z} \text { for all } \eta \in D\right\} .
$$

We say that $\widehat{D^{\prime} \mid D}$ is the dual of $D$ with respect to $D^{\prime}$. The following result is obvious.

LEMMA 1.12. $\widehat{D^{\prime} \mid D}=\left(\beta\left(D^{\prime}\right) / \beta(D)\right) \hat{D}$.

We want to investigate the relationship between $D, D^{\prime}$ and $\widehat{D^{\prime} \mid D}$. A useful fact is the next lemma.

LEMma 1.13. For $D \in H \mathcal{E}\left(C^{n}\right)$ with $D \subset D_{n}$,

$$
O\left(D \backslash D_{n}\right)=\beta(D)^{n} \cdot \mathscr{P}(D)^{1 / 2} \text {. }
$$

Proof. Write $D=d \cdot \alpha(D(l))$ as above. Then $D=T\left(D_{n}\right)$ where 


$$
T=d \cdot \alpha \cdot\left(\begin{array}{ccccc}
E_{n} & & & & \\
& 1 & & & \\
& & l_{2} & & \\
& & & \ddots & \\
0 & & & & l_{n}
\end{array}\right) .
$$

However, det $T=d^{2 n} \mathscr{P}(D)^{1 / 2}$. Since $\beta(D)=d^{2}$, we are done.

Lemma 1.14. Let $D \subset D^{\prime}$ be two lattices in $H \mathcal{L}\left(C^{n}\right)$. Then

$$
\begin{gathered}
O\left(D \backslash D^{\prime}\right)=\left(\beta(D) / \beta\left(D^{\prime}\right)\right)^{n}\left(\mathcal{P}(D) / \mathcal{P}\left(D^{\prime}\right)\right)^{1 / 2} ; \\
O\left(\widehat{D^{\prime} \mid D} / D^{\prime}\right)=O\left(D \backslash D^{\prime}\right) \cdot \mathcal{P}\left(D^{\prime}\right) .
\end{gathered}
$$

Proof. Write $D^{\prime}-d^{\prime} \cdot \alpha\left(D\left(l^{\prime}\right)\right)$ as above. Then $D^{\prime \prime}=\left(1 / d^{\prime}\right) \alpha^{-1}(D) \subset$ $D\left(l^{\prime}\right) \subset D_{n}$. Since

$$
O\left(D_{n} / D\left(l^{\prime}\right)\right)=\mathscr{P}\left(D^{\prime}\right)^{1 / 2}
$$

and

$$
O\left(D_{n} / D^{\prime \prime}\right)=\beta\left(D^{\prime}\right)^{n} \mathcal{P}\left(D^{\prime \prime}\right)^{1 / 2}=\left(\beta(D) / \beta\left(D^{\prime}\right)\right)^{n} \mathscr{P}(D)^{1 / 2},
$$

we have the first result.

The second result immediately follows from Lemmas 1.11 and 1.12 in view of (a).

The above results translate immediately into a duality theory for $\mathcal{L}$. Since each $D \in H \mathcal{L}\left(\mathrm{C}^{n}\right)$ determines a $\Gamma \in \mathcal{L}$ only up to conjugation in $N_{n}$, what is really considered is a duality theory of conjugacy classes of $\mathcal{L}$.

Let $\Gamma \in \mathcal{L}$ and set $D=\pi \Gamma$. Choose a lattice $\Gamma^{\prime}$ in $N_{n}$ such that $\pi \Gamma^{\prime}=\hat{D}$. As we have shown, $\Gamma^{\prime}$ is determined up to inner automorphism in $N_{n}$. We denote the conjugacy class containing $\Gamma^{\prime}$ as an element by $\hat{\Gamma}$. Also, we can also choose a $\Gamma^{\prime} \in \hat{\Gamma}$ so that $\Gamma \subset \Gamma^{\prime}$. By abuse of language we shall also consider $\hat{\Gamma}$ as a lattice itself satisfying $\Gamma \subset \hat{\Gamma}$.

Lemma 1.15. For $\Gamma \in \mathcal{L}, O(\Gamma[\hat{\Gamma}, \hat{\Gamma}] \backslash \hat{\Gamma})=\mathscr{P}(\Gamma)$.

Suppose $\Gamma, \Gamma^{\prime} \in \mathcal{L}$ and $\Gamma \subset \Gamma^{\prime}$. Set $D=\pi(\Gamma)$ and $D^{\prime}=\pi\left(\Gamma^{\prime}\right)$. Choose a lattice $\Gamma^{\prime \prime}$ satisfying $\pi \Gamma^{\prime \prime}=\widehat{D^{\prime} \mid D}$. The conjugacy class containing $\Gamma^{\prime \prime}$ is denoted by $\widehat{\Gamma^{\prime}} \mid \Gamma$, and, as above, $\widehat{\Gamma \mid \Gamma}$ will also denote a lattice contained in $\widehat{\Gamma^{\prime} \mid \Gamma}$ as a conjugacy class satisfying $\Gamma \subset \Gamma^{\prime} \subset \widehat{\Gamma^{\prime} \mid \Gamma}$.

Lemma 1.16. Let $\Gamma \subset \Gamma^{\prime}$ be lattices in $N_{n}$. Then

$$
\widehat{\Gamma^{\prime} \mid \Gamma}=\left(\beta\left(\Gamma^{\prime}\right) / \beta(\Gamma)\right) \hat{\Gamma}
$$

where 


$$
d \cdot \gamma=\left(\begin{array}{cc}
d E_{2 n} & 0 \\
0 & d^{2}
\end{array}\right) \cdot \gamma
$$

when $d \in R$ and $\gamma \in N_{n}$.

Proof. Directly from definition.

LEMMA 1.17. For $\Gamma \subset \Gamma^{\prime}$ lattices in $N_{n}$ the following results hold.

(a) If $\Gamma \subset \Gamma_{n}$, then

$$
O\left(\Gamma \backslash \Gamma_{n}\right)=\beta(\Gamma)^{n} \mathscr{P}(\Gamma)^{1 / 2}
$$

$$
\begin{gathered}
O\left(\Gamma\left[\Gamma^{\prime}, \Gamma^{\prime}\right] \backslash \Gamma^{\prime}\right)=\left(\beta(\Gamma) / \beta\left(\Gamma^{\prime}\right)\right)^{n}\left(\mathscr{P}(\Gamma) / \mathscr{P}\left(\Gamma^{\prime}\right)\right)^{1 / 2} . \\
O\left(\widehat{\Gamma^{\prime} \mid \Gamma} / \Gamma^{\prime}\left[\widehat{\Gamma^{\prime} \mid \Gamma}, \widehat{\Gamma^{\prime} \mid \Gamma}\right]\right)=O\left(\Gamma^{\prime} / \Gamma\left[\Gamma^{\prime}, \Gamma^{\prime}\right]\right) \cdot \mathscr{P}\left(\Gamma^{\prime}\right) .
\end{gathered}
$$

Proof. Follows from the corresponding results for lattices in $\mathrm{C}^{n}$.

2. Analysis. Let $\Gamma$ be a lattice in $N_{n}$. The space of right cosets $\Gamma \backslash N_{n}=$ $\left\{\Gamma v: v \in N_{n}\right\}$ admits a unique probability measure invariant under translations by $N_{n}$. This measure will be assumed in all that follows. Hence, we get a unitary representation $R$ of $N_{n}$ of $\mathcal{L}^{2}\left(\Gamma \backslash N_{n}\right)$ by setting

$$
R_{v}(F(\Gamma w))=F(\Gamma w v), \quad F \in \mathcal{L}^{2}\left(\Gamma \backslash N_{n}\right), \quad v, w \in N_{n} .
$$

We will write functions $F$ on $\Gamma \backslash N_{n}$ as functions on $N_{n}$ which are fixed under left translations by $\Gamma$. In general, if $F$ is a function on $N_{n}$ and $v \in N_{n}$ we set

$$
L_{v} F(w)=F\left(v^{-1} w\right), \quad w \in N_{n} \text {. }
$$

Thus, the condition on a function $F$ to be a function on $\Gamma \backslash N_{n}$ is given by $L_{\gamma} F=F$ for all $\gamma \in \Gamma$. We will refer to such functions as $\Gamma$-periodic functions.

The beginning of this section recalls some of the basic results concerning the representation $R$. In particular, we reconsider the distinguished subspace theory of L. Auslander and J. Brezin. Analogous to $\S 1$, the central theme is how the automorphism group and the duality theory controls the structure of the distinguished subspaces. Essentially, once we know the multiplicity space $H_{1}(\Gamma)$, defined below, the theory of distinguished subspaces can be derived algebraically. The next topic is the construction of a special function theory for $\Gamma \backslash N_{n}$. The guiding force in this theory is the concept of CR-structures. H. Rossi, in 1970, was apparently the first to see how CR-structures played an important role in developing a special function theory. Since both the distinguished subspace theory and the special function theory are built out of the automorphism group, it comes as no surprise that they are intimately related. This relationship is exploited to give an analytic characterization of the distinguished subspaces (see [1] where this result first appears). The final topic is the application of our theory to classical theta function theory.

For each $m \in Z$ put 


$$
H_{m}(\Gamma)=\left\{F \in \mathcal{L}^{2}\left(\Gamma \backslash N_{n}\right): F(\xi, z)=e^{2 \pi i m z / \beta(\Gamma)} F(\xi, 0)\right\} .
$$

Each $H_{m}(\Gamma)$ is easily seen to be $R$-invariant and we have the $\mathfrak{L}^{2}$-decomposition

$$
\mathscr{L}^{2}\left(\Gamma \backslash N_{n}\right)=\sum_{m \in Z} \oplus H_{m}(\Gamma)
$$

Moreover, for each $F \in \mathcal{L}^{2}\left(\Gamma \backslash N_{n}\right)$ the projection $p_{m}(F)$ of $F$ into $H_{m}(\Gamma)$ is given by the formula

$$
p_{m} F(\xi, z)=\int_{0}^{\beta(T)} e^{-2 \pi i(m \cdot s) / \beta(\Gamma)} F(\xi, z+s) d s .
$$

The Stone-von Neumann theorem implies $H_{1}(\Gamma)$ is $R$-invariant and irreducible, and any two $R$-invariant and irreducible subspaces $g_{1}$ and $g_{2}$ of $\mathcal{L}^{2}\left(\Gamma \backslash N_{n}\right)$ are unitarily equivalent if and only if $g_{1}, g_{2} \subset H_{m}(\Gamma)$ for some $m \in Z$. We assume this result in what follows. Clearly, when $m=0, H_{0}(\Gamma \backslash$ $\left.N_{n}\right)=\mathcal{L}^{2}\left(\pi \Gamma \backslash R^{2 n}\right)$ and $H_{0}\left(\Gamma \backslash N_{n}\right)$ decomposes into the infinite $\mathcal{L}^{2}$-direct sum of 1-dimensional $R$-invariant subspaces. Below we will show that $R \mid H_{m}(\Gamma)$ is the product of $|m|^{n} \mathscr{P}(\Gamma)$ copies of a single irreducible representation of $N_{n}$ when $m \neq 0$.

Let $\Gamma$ be a lattice in $N_{n}$. For any automorphism $\alpha \in \mathbb{Q}_{n}^{0}$ it is directly verified that the following results hold.

(6)(a) $\mathcal{L}^{2}\left(\Gamma \backslash N_{n}\right) \cdot \alpha^{-1}=\mathcal{L}^{2}\left(\Gamma^{\alpha} \backslash N_{n}\right)$.

(b) $H_{m}(\Gamma) \cdot \alpha^{-1}=H_{m}\left(\Gamma^{\alpha}\right)$.

(c) If $\mathcal{G}$ is an $R$-invariant and irreducible subspace of $\mathcal{L}^{2}\left(\Gamma \backslash N_{n}\right)$ then $g \cdot \alpha^{-1}$ is an $R$-invariant and irreducible subspace of $\mathcal{L}^{2}\left(\Gamma^{\alpha} \backslash N_{n}\right)$.

Thus, knowing $R$ on $\mathcal{L}^{2}\left(\Gamma \backslash N_{n}\right)$ is equivalent to knowing $R$ on $\mathscr{L}^{2}\left(\Gamma^{\alpha} \backslash N_{n}\right)$. For this reason it is sufficient as far as representation theory is concerned to restrict our attention to those lattices $\Gamma=\Gamma(l), l \in Z_{n}^{\#}$.

Let $\Gamma=\Gamma(l)$. Then $\Gamma \subset \Gamma_{n}$ and choose $\Gamma^{\prime}=\widehat{\Gamma_{n} \mid \Gamma}$ to contain $\Gamma_{n}$. As we have said, $\Gamma^{\prime}$ is a maximal element from the class of lattices in $N_{n}$ having the property that $\left[\Gamma^{\prime}, \Gamma^{\prime}\right] \subset \Gamma_{n}$. It follows by elementary arguments that each $\gamma^{\prime} \in \Gamma^{\prime}$ determines a character $\chi\left(\gamma^{\prime}\right)$ on $\Gamma_{n}$ by the rule

$$
\chi\left(\gamma^{\prime}\right)(\gamma)=e^{2 \pi i}\left[\gamma, \gamma^{\prime}\right], \quad \gamma \in \Gamma_{n} .
$$

Moreover, the mapping $\gamma^{\prime} \mapsto \chi\left(\gamma^{\prime}\right)$ induces an isomorphism

$$
\Gamma_{n}\left[\Gamma^{\prime}, \Gamma^{\prime}\right] \backslash \Gamma^{\prime} \cong \operatorname{ch}\left(\Gamma \backslash \Gamma_{n}\right) \text {. }
$$

We denote the abstract group in (8) by $\Delta=\Delta(\Gamma)$, where in context it will be clear which of the two groups is meant.

LEMMA 2.1. For each $\gamma^{\prime} \in \Gamma, L_{\gamma^{\prime}} H_{1}\left(\Gamma_{n}\right)$ is an $R$-invariant and irreducible subspace of $H_{1}(\Gamma)$ and can be given by

$$
L_{\gamma^{\prime}} H_{1}\left(\Gamma_{n}\right)=\left\{F \in H_{1}(\Gamma): L_{\gamma} F=\chi\left(\gamma^{\prime}\right)(\gamma) F \text { for all } \gamma \in \Gamma_{n}\right\} \text {. }
$$


Proof. Since $L_{\gamma^{\prime}} R_{v}=R_{v} L_{\gamma^{\prime}}$ and $H_{1}\left(\Gamma_{n}\right)$ is $R$-invariant and irreducible, the first part of the lemma is proved once we know $L_{\gamma} H_{1}\left(\Gamma_{n}\right) \subset H_{1}(\Gamma)$. This follows from $\left[\Gamma^{\prime}, \Gamma\right] \subset \Gamma_{n}$. To prove the second part of the lemma, first observe that $H_{1}\left(\Gamma_{n}\right)$ is the eigenvalue one space in $H_{1}(\Gamma)$ with respect to the action of $\Gamma_{n}$ on $H_{1}(\Gamma)$ by left-translation. Take $F \in H_{1}\left(\Gamma_{n}\right)$ and $\gamma^{\prime} \in \Gamma^{\prime}$. Then

$$
L_{\gamma}\left(L_{\gamma^{\prime}} F\right)(v)=F\left(\gamma^{\prime-1} \gamma^{-1} v\right)=F\left(\left[\gamma^{\prime}, \gamma\right] \gamma^{-1} \gamma^{\prime-1} v\right)=e^{\left.2 \pi i \gamma^{\prime}, \gamma\right]} L_{\gamma^{\prime}} F(v)
$$

for all $\gamma \in \Gamma_{n}$. Conversely, if $G \in H_{1}(\Gamma)$ satisfies $L_{\gamma} G=\chi\left(\gamma^{\prime}\right)(\gamma) G$ for all $\gamma \in \Gamma_{n}$ then $L_{\gamma^{\prime-1}} G \in H_{1}\left(\Gamma_{n}\right)$. Hence, we have proved the lemma.

COROLlary. For each $\gamma^{\prime} \in \Gamma^{\prime}, H_{1}\left(\Gamma_{n}\right)$ and $L_{\gamma^{\prime}} H_{1}\left(\Gamma_{n}\right)$ are unitarily equivalent.

Proof. Follows from the first sentence of the proof of the lemma.

THEOREM 2.2. Let $\Gamma=\Gamma(l)$. Then

$$
H_{1}(\Gamma)=\sum_{\gamma^{\prime} \in \Delta(\Gamma)} \oplus L_{\gamma^{\prime}} H_{1}\left(\Gamma_{n}\right)
$$

is a direct sum decomposition of $H_{1}(\Gamma)$ into $R$-invariant, irreducible and unitarily equivalent subspaces.

Proof. The group $\Gamma \backslash \Gamma_{n}$ acts on $H_{1}(\Gamma)$ by left-translations and hence decomposes $H_{1}(\Gamma)$ into subspaces determined by $\operatorname{ch}\left(\Gamma \backslash \Gamma_{n}\right)$. By (8) and Lemma 2.1 all the characters appear and we are done.

COROLlary. Let $\Gamma$ be an arbitrary lattice in $N_{n}$. Then $R \mid H_{1}(\Gamma)=$ the restriction of $R$ to $H_{1}(\Gamma)$ is $\mathscr{P}(\Gamma)^{1 / 2}$ times a single irreducible representation of $N_{n}$.

Proof. Simply translate by (6) Theorem 2.2 .

We will now develop distinguished subspace theory from the point of view of the automorphism group. Define for a lattice $\Gamma=\Gamma(l)$ and an integer $m>0$,

$$
B_{m}(\Gamma)=\left\{\alpha \in \mathbb{Q}_{n}^{0}: \alpha(\Gamma) \subset \Gamma \text { and } \alpha \mid Z=m \cdot E_{1}\right\},
$$

where $E_{1}$ denotes the identity mapping of $Z$.

We will associate to each $\alpha \in B_{m}(\Gamma)$ an $\mathscr{L}^{2}$-decomposition of $H_{m}(\Gamma)$ into $R$-invariant and irreducible subspaces.

Set $\Gamma=\Gamma(l), l \in Z_{n}^{\#}$, and define

$$
\Gamma(m)=\operatorname{gp}\left\{\Gamma, m^{-1}[\Gamma, \Gamma]\right\}, \quad m \in Z_{+}^{x} .
$$

To each $\alpha \in B_{m}(\Gamma), \Gamma^{\alpha}=\alpha \Gamma \subset \Gamma \subset \Gamma_{n}$. Choose $\Gamma^{*}=\widehat{\Gamma_{n} \mid \Gamma^{\alpha}}$ containing $\Gamma_{n}$. To each $\gamma^{*} \in \Gamma^{*}$ we associate the character $\chi\left(\gamma^{*}\right)$ of $\Gamma_{n}^{\alpha-1}$ defined by the rule

$$
\chi\left(\gamma^{*}\right)\left(\alpha^{-1}(\gamma)\right)=e^{2 \pi i m\left[\gamma^{*}, \alpha^{-1}(\gamma)\right]}, \quad \gamma \in \Gamma_{n} .
$$


An elementary exercise shows that the mapping $\gamma^{*} \mapsto \chi\left(\gamma^{*}\right)$ induces the following isomorphism.

$$
\Gamma_{n}\left[\Gamma^{*}, \Gamma^{*}\right] \backslash \Gamma^{*} \cong \operatorname{ch}\left(\Gamma(m) \backslash \Gamma_{n}^{\alpha^{-1}}\right) \text {. }
$$

LEMMA 2.3. To each $\gamma^{*} \in \Gamma^{*}, L_{\gamma^{*}}\left(H_{1}\left(\Gamma_{n}\right) \cdot \alpha\right)$ is an $R$-invariant and irreducible subspace of $H_{m}(\Gamma)$ and is given by

$$
\begin{aligned}
L_{\gamma^{*}} & \left(H_{1}\left(\Gamma_{n}\right) \cdot \alpha\right) \\
& =\left\{F \in H_{m}(\Gamma): L_{\alpha^{-1}(\gamma)} F=\chi\left(\gamma^{*}\right)\left(\alpha^{-1}(\gamma)\right) F \text { for all } \gamma \in \Gamma_{n}\right\} .
\end{aligned}
$$

Proof. The first statement follows as Lemma 2.1 using $\left[\Gamma^{*}, \Gamma\right] \subset \Gamma_{n}(m)$. The second statement follows as Lemma 2.1.

COROLlaRY. For each $\gamma^{*} \in \Gamma^{*}, L_{\gamma^{*}}\left(H_{1}\left(\Gamma_{n}\right) \cdot \alpha\right)$ is unitarily equivalent to $H_{1}\left(\Gamma_{n}\right) \cdot \alpha$.

THEOREM 2.4. For each lattice $\Gamma=\Gamma(l), \alpha \in B_{m}(\Gamma)$,

$$
H_{m}(\Gamma)=\sum_{\gamma^{*}} \oplus L_{\gamma^{*}}\left(H_{1}\left(\Gamma_{n}\right) \cdot \alpha\right)
$$

where $\gamma^{*}$ runs over $\Gamma_{n}\left[\Gamma^{*}, \Gamma^{*}\right] \backslash \Gamma^{*}, \Gamma^{*}=\widehat{\Gamma_{n} \mid \Gamma^{\alpha}}$.

Proof. $\Gamma(m) \backslash \Gamma_{n}^{\alpha^{-1}}$ is a finite group and acts on $H_{m}(\Gamma)$ by left-translation having $H_{1}\left(\Gamma_{n}\right) \cdot \alpha$ as its eigenvalue one space. This action decomposes $H_{m}(\Gamma)$ into an $\mathfrak{L}^{2}$-direct sum (see $[1$, p. 8]) corresponding to characters on $\Gamma(m) \backslash$ $\Gamma_{n}^{\alpha^{-1}}$, all of which occur nontrivially by (13) and Lemma 2.3.

COROllary. $R \mid H_{m}(\Gamma)$ is $|m|^{n} \mathscr{P}(\Gamma)^{1 / 2}$ times a single irreducible representation of $N_{n}$.

Proof. Combine Lemma 2.3, its corollary and Theorem 2.4 along with $O\left(\Gamma_{n}\left[\Gamma^{*}, \Gamma^{*}\right] \backslash \Gamma^{*}\right)=|m|^{n} \mathcal{P}(\Gamma)^{1 / 2}$.

We will now use the theory of CR-structures discussed in $\$ 1$ to pick out a special subspace of functions in $N_{n}$. Essentially, we mimic the way holomorphic functions on $\mathrm{C}^{n}$ are determined by the Cauchy-Riemann equations. To do so we must first describe the Lie theoretic language which allows this translation. Recall, $L_{n}=$ Lie algebra of $N_{n}$. Set $L_{n}^{\mathrm{C}}=$ the complexification of $L_{n}$, which we shall regard as a left-invariant differential operator on $\mathrm{C}^{\infty}\left(N_{n}\right)$. The vectors

$$
\begin{array}{ll}
X_{j}=\partial / \partial x_{j}+\left(y_{j} / 2\right) \partial / \partial z, & 1 \leqslant j \leqslant n, \\
Y_{j}=\partial / \partial y_{j}-\left(x_{j} / 2\right) \partial / \partial z, & 1<j<n, \\
Z=\partial / \partial z &
\end{array}
$$

determine a C-basis of $L_{n}^{\mathrm{C}}$. The action of $\mathbb{Q}_{n}^{0}$ on $L_{n}^{\mathrm{C}}$ is given as follows. For $\boldsymbol{\xi}=(\mathbf{x}, \mathbf{y}) \in \mathrm{C}^{2 n}$ and $\mathbf{z} \in \mathrm{C}$, put 


$$
(\xi, z)=\mathbf{x}^{\prime} \cdot \mathbf{X}+\mathbf{y}^{t} \mathbf{Y}+\mathbf{z Z}=\sum \mathbf{x}_{j} X_{j}+\sum \mathbf{y}_{j} Y_{j}+\mathbf{z Z}
$$

where

$$
\mathbf{x}=\left(\mathbf{x}_{1}, \ldots, \mathbf{x}_{n}\right) \in \mathrm{C}^{n}, \quad \mathbf{X}=\left(\begin{array}{c}
X_{1} \\
\vdots \\
X_{n}
\end{array}\right)
$$

and corresponding notation for $y$ 's. Let

$$
\alpha=\left(\begin{array}{cc}
S & 0 \\
\delta^{\prime} & d
\end{array}\right)
$$

$d>0$, be in $\mathbb{E}_{n}^{0}$. Then

$$
\alpha(\xi, \mathbf{z})=\left(S \xi, d \mathbf{z}+\delta^{t} \cdot \xi\right) .
$$

In particular, we will apply (16) to positive definite CR-structures. Let $\beta$ be a positive definite CR-structure. Then $\beta$ determines a direct sum decomposition of $L_{n}^{\mathrm{C}}$,

$$
L_{n}^{\mathrm{C}}=V_{i}(\beta) \oplus V_{-i}(\beta) \oplus Z_{\mathrm{C}},
$$

where $V_{ \pm i}(\beta)$ is the eigenvalue $\pm i$ subspace of $L_{n}^{C}$ with respect to $\beta$ and $Z_{\mathrm{C}}=$ center of $L_{n}^{\mathrm{C}}$. Clearly, $\overline{V_{i}(\beta)}=V_{-i}(\beta)$. For reasons which will be made apparent below we will be solely concerned with the space $V_{i}(\beta)$.

Define

$$
\theta(\beta)=\left\{G \in C^{\infty}\left(N_{n}\right): V_{i}(\beta) G=0\right\} .
$$

We call a function $G$ a $\beta$-theta function whenever $G \in \theta(\beta)$. The automorphism group $\mathbb{Q}_{n}^{0}$ relates the various subspaces of $\beta$-theta functions. Firstly, we can write $\beta=\alpha^{-1} J_{2 n} \alpha$ where $\alpha \in \mathbb{Q}_{n}^{0}$. The choice of $\alpha$ is, of course, not unique but is determined uniquely up to left multiplication by an element from $\theta(2 n, R)$ and multiplication by elements from $\mathscr{D}_{2 n}$. Obviously,

$$
V_{i}(\beta)=\alpha^{-1}\left(V_{i}\left(J_{2 n}\right)\right), \quad \theta(\beta)=\theta\left(J_{2 n}\right) \cdot \alpha .
$$

The first equality is purely formal while the second follows by the chain rule.

We want an explicit basis for $V_{i}(\beta)$. We begin with $V_{i}\left(J_{2 n}\right)$. Let $\mathbf{v}=(\xi, \mathbf{z})$ $\in V_{i}\left(J_{2 n}\right), \xi=(\mathbf{x}, \mathbf{y})$. It follows that $J_{2 n}(\mathrm{v})=i \mathrm{v}$ which implies $\mathbf{y}=i \mathbf{x}$ and $\mathrm{z}=0$. Thus, the $n$ vectors

$$
\mathbf{X}+i \mathbf{Y}
$$

define a C-basis of $V_{i}\left(J_{2 n}\right)$. Let

$$
\beta=\left(\begin{array}{cc}
S & 0 \\
\gamma^{\prime} & 1
\end{array}\right)
$$

be an arbitrary positive definite CR-structure where $S=\left(\begin{array}{cc}A & B \\ C & D\end{array}\right)$ and $\gamma=\left(\gamma^{\prime}\right.$, 
$\left.\gamma^{\prime \prime}\right) \in R^{2 n}$. We want to describe a basis of $V_{i}(\beta)$ in terms of $S$ and $\gamma$. For $\mathbf{v}=(\xi, \mathbf{z}) \in V_{i}(\beta), \beta(\mathbf{v})=i \mathrm{v}$ implies

$$
\mathbf{z}=\left(\frac{i+1}{2}\right) \gamma^{\prime} \cdot \xi \text { and } \mathbf{y}=B^{-1}\left(i E_{n}-A\right) \mathbf{x}=\left(i E_{n}-D\right)^{-1} C \mathbf{x} \text {. }
$$

Our previous characterization of positive definite CR-structures allows the taking of inverses. Hence, the $n$ vectors

$$
\mathbf{X}+i G(\beta) \mathbf{Y}+((i+1) / \sqrt{2})\left(\gamma^{\prime}+i \gamma^{\prime \prime}\right) \mathbf{Z}
$$

where $G(\beta)=B^{-1}\left(i A+E_{n}\right)$, define a $C$-basis of $V_{i}(\beta)$. Observe that $B$ is symmetric and positive definite and $A B$ is symmetric, implying that $G(\beta)$ is symmetric and has a positive definite real part.

For a positive definite CR-structure $\beta$ we can write $\beta=\alpha^{-1} J_{2 n} \alpha, \alpha \in \mathbb{Q}_{n}^{0}$. We want to write (21) in terms of $\alpha$. Firstly, we will digress to give an interpretation of $\mathbb{Q}_{n}^{0}\left[J_{2 n}\right]=\left\{\alpha^{-1} J_{2 n} \alpha: \alpha \in \mathbb{Q}_{n}^{0}\right\}$ which put our eventual formulas into their proper context. Let $3_{n}$ be the space of $n \times n$ complex symmetric matrices $T=X+i Y$ where $T$ is positive definite. The space $B_{n}$ is called the $n$-dimensional Siegel upper half plane. $\operatorname{Sp}(2 n, R)$ acts on $3_{n}$ under fractional linear transformations defined by the rule

$$
(T) M=(A+T C)^{-1}(B+T D),
$$

where $M=\left(\begin{array}{cc}A & B \\ C & D\end{array}\right) \in \operatorname{Sp}(2 n, R), T \in 3_{n}$. A standard fact is $(T) M \in 3_{n}$ whenever $T \in 3_{n}$. Also, $\operatorname{Sp}(2 n, R)$ acts transitively on $Z_{n}$ and, hence, the mapping $M \mapsto\left(i E_{n}\right) M$ induces a bijection between $K \backslash \operatorname{Sp}(2 n, R)$ and $Z_{n}$, where $K=\operatorname{Sp}(2 n, R) \cap \mathcal{\theta}(2 n, R)$. Recall, we have set $\mathscr{P}_{2 n}^{*}=\operatorname{Sp}(2 n, R)\left[J_{2 n}\right]$, and it follows immediately that the mapping $M \mapsto M^{-1} J_{2 n} M$ induces a bijection between $K \backslash \operatorname{Sp}(2 n, R)$ and $\mathscr{P}_{2 n}^{*}$.

LEMMA 2.5. Let $\beta=M^{-1} J_{2 n} M, M \in \operatorname{Sp}(2 n, R)$. Then $i G(\beta)^{-1}=\left(i E_{n}\right) M$.

Proof. Setting

$$
M=\left(\begin{array}{ll}
A_{1} & B_{1} \\
C_{1} & D_{1}
\end{array}\right) \text { and } \beta=\left(\begin{array}{ll}
A & B \\
C & D
\end{array}\right),
$$

then

$$
C=-\left(A_{1}^{t}-i C_{1}^{t}\right)\left(A_{1}+i C_{1}\right), \quad B=\left(D_{1}^{t}+i B_{1}^{t}\right)\left(D_{1}-i B_{1}\right)
$$

and

$$
i A+E_{n}=\left(D_{1}^{t}+i B_{1}^{t}\right)\left(A_{1}+i C_{1}\right) \text {. }
$$

The lemma follows from (21) and (22).

An important property about $\theta(\beta)$ which follows directly by the chain rule is asserted in the next lemma.

LEMMA 2.6. $L_{v} \theta(\beta)=\theta(\beta)$ for all $\beta \in \mathscr{P}_{2 n}$ and $v \in N_{n}$. 
We are mainly interested in those functions $F \in \theta(\beta)$ which are $\Gamma$-periodic where $\Gamma$ is a lattice. For $\beta \in \mathscr{P}_{2 n}$ and $\Gamma \in \mathcal{L}$ define

$$
\theta(\beta, \Gamma)=\theta(\beta) \cap \mathcal{L}^{2}\left(\Gamma \backslash N_{n}\right), \theta_{m}(\beta, \Gamma)=\theta(\beta) \cap H_{m}(\Gamma) .
$$

We will eventually describe these spaces in terms of $\beta$ and $\Gamma$.

Lemma 2.7. For $F \in \theta(\beta, \Gamma)$, the projection $p_{m}(F)$ into $H_{m}(\Gamma)$ is in $\theta_{m}(\beta, \Gamma)$.

Proof. It is straightforward to verify that

$$
V_{i}(\beta) p_{m}(F)(\xi, z)=\int_{0}^{\beta(\Gamma)} e^{-2 \pi i(m \cdot s) / \beta(\Gamma)} V_{i}(\beta) F(\xi, z+s) d s,
$$

which immediately implies the lemma.

Corollary. $\theta(\beta, \Gamma)=\Sigma_{m \in Z} \oplus \theta_{m}(\beta, \Gamma)$ in the $\mathcal{L}^{2}$-norm.

Again, the automorphism group will be utilized to relate the various spaces. Suppose $\beta \in \mathscr{P}_{2 n}$ and $\Gamma \in \mathcal{L}$. Then $\beta=\alpha^{-1} J_{2 n} \alpha, \alpha \in \mathbb{Q}_{n}^{0}$, and $\Gamma=\gamma(\Gamma(l)$ ), where $\gamma \in \mathbb{Q}_{n}^{0}$ and $l \in Z_{n}^{\#}$. Then

$$
\theta(\beta, \Gamma)=\theta\left(\gamma \alpha^{-1} J_{2 n} \alpha \gamma^{-1}, \Gamma(l)\right) \cdot \gamma^{-1} \text {. }
$$

The first use we make of (24) is to reduce the problem of computing the subspaces $\theta(\beta, \Gamma)$ to the special case where $\Gamma=\Gamma(l)$ and $\beta$ is arbitrary or the special case where $\Gamma$ is arbitrary and $\beta=J_{2 n}$.

LEMMA 2.8. $\theta_{0}(\beta, \Gamma)=C$.

Proof. Assume $\beta=J_{2 n}$. Then if $F \in \theta_{0}(\beta, \Gamma)$ it is easy to see that $F(\xi)$, $\xi=x+i y \in C^{n}$, is entire. Since it is also periodic, it must be a constant.

Before we consider $\theta_{m}(\beta, \Gamma)$ for $m>0$ we remark on the following description of $H_{1}\left(\Gamma_{n}\right)$. Each $F \in H_{1}\left(\Gamma_{n}\right)$ can be written

$$
F(\xi, z)=e^{2 \pi i z} e^{+\pi i x \cdot y} F_{1}(\xi), \quad \xi=(x, y),
$$

where

$$
F_{1}(\xi)=\sum_{l \in Z^{n}} f(y+l) e^{2 \pi i l x} \text { and } f(y)=\int_{0}^{E_{n}} F_{1}(\xi) d x .
$$

The function $F_{1}(\xi)$ will be denoted by $P(f)(\xi)$ to show its dependence on $f$. Clearly, $f \in \mathscr{L}^{2}\left(R^{n}\right)$ and is not uniquely determined by the condition $F=$ $e^{2 \pi i z} e^{-\pi i x \cdot y} P(f)$.

For

$$
\beta=\left(\begin{array}{cc}
S & 0 \\
\gamma^{\prime} & 1
\end{array}\right) \in \mathscr{P}_{2 n}
$$

define the function on $N_{n}$ by the rule 


$$
\psi\left(\beta, \Gamma_{n}\right)(\xi, z)=e^{2 \pi i z} e^{-\pi i x \cdot y} P(f)(\xi),
$$

where

$$
f(y)=\exp \left\{\pi i\left(i G(\beta)^{-1}[y]+(i-1) G(\beta)^{-1}\left(\gamma^{\prime}+i \gamma^{\prime \prime}\right) \cdot y\right)\right\} .
$$

THEOREM 2.9. $\theta_{1}\left(\beta, \Gamma_{n}\right)$ is a 1-dimensional $\mathrm{C}$-vector space and the function $\psi\left(\beta, \Gamma_{n}\right)$ exists and is in $\theta_{1}\left(\beta, \Gamma_{n}\right)$.

Proof. The real part of $G(\beta)$ being symmetric and positive definite immediately implies the function in (26) exists and hence is in $H_{1}\left(\Gamma_{n}\right)$. We shall now show that it is, in fact, up to constant multiple the unique function in $\theta_{1}\left(\beta, \Gamma_{n}\right)$. Assume $F \in \theta_{1}\left(\beta, \Gamma_{n}\right)$. Since $F \in C^{\infty}\left(N_{n}\right)$ it follows $P(f)$ is also infinitely differentiable. Hence, $f(y)=\int_{0}^{E_{n}} P(f) d x$ is infinitely differentiable and its derivatives may be computed by differentiating inside the integral. Using $\mathbf{Y}\left(e^{2 \pi i z} e^{+\pi i x \cdot y}\right)=0$, condition (21) becomes

$$
i G(\beta) \mathbf{Y} P(f)=-2 \pi i y P(f)-\mathbf{X} P(f)+(i+1) \pi i\left(\gamma^{\prime}+i \gamma^{\prime \prime}\right) P(f) .
$$

However, $\int_{0_{n}}^{E_{n}} \mathbf{X} P(f) d x=0$ by periodicity in $x$. Hence,

$$
i G(\beta) \partial f(y) / \partial y=\left(-2 \pi i y+(i+1) \pi i\left(\gamma^{\prime}+i \gamma^{\prime \prime}\right)\right) f(y),
$$

and, as is standard knowledge, this equation has a unique solution, up to constant multiple, given by the formula in (26).

Corollary. Let $\Gamma=\Gamma(l)$ and $\alpha \in B_{m}(\Gamma), m>0$. Then

$$
\theta_{m}(\beta, \Gamma)=\sum_{\gamma^{*}} L_{\gamma^{*}}\left(\theta\left(\alpha \beta \alpha^{-1}, \Gamma_{n}\right) \cdot \alpha\right)
$$

where $\gamma^{*}$ is as in Theorem 2.4. In particular,

$$
\operatorname{dim}_{\mathrm{C}} \theta_{m}(\beta, \Gamma)=m^{n} \mathscr{P}(\Gamma) .
$$

Proof. Simply apply Lemma 2.7, Theorem 2.4 and (19) to the previous theorem.

Corollary. To each distinguished subspace $g$ of $H_{m}\left(\Gamma_{n}\right)$,

$$
\operatorname{dim}_{C}\left(g \cap \theta_{m}\left(\beta, \Gamma_{n}\right)\right)=\mathcal{P}\left(\Gamma_{n}\right)=1 .
$$

Before continuing we will make several observations about these results. Firstly, although we gave an explicit function $\psi_{1}\left(\beta, \Gamma_{n}\right)$ to describe $\theta_{1}\left(\beta, \Gamma_{n}\right)$, this function is, in fact, explicitly associated to the pair $\left(\beta, \Gamma_{n}\right)$. Also, the question of convergence, i.e. uniform convergence on compact subsets of the series in (26), immediately indicates $\theta_{m}\left(\beta, \Gamma_{n}\right)=0$ whenever $m<0$.

Thus far we have proceeded by describing the function theory related to a fixed choice of a lattice in $N_{n}$. The final topic of this section involves the following general question. What information does the function theory give us about the lattices in $N_{n}$ ? 
LEMMA 2.10. Let $\Gamma$ be a lattice in $N_{n}$ and choose $\beta \in \mathscr{P}_{2 n}$. Then

$$
\Gamma=\left\{v \in N_{n}: L_{v} F=F \text { whenever } F \in \theta_{1}(\beta, \Gamma)\right\} \text {. }
$$

Proof. Suppose to begin with $\Gamma=\Gamma_{n}$. Then it is easy to see by direct computation that $\psi_{1}\left(\beta, \Gamma_{n}\right)$ can be fixed by $L_{v}, v \in N_{n}$, if and only if $v \in \Gamma_{n}$. For $\Gamma=\Gamma(l)$ we consider the functions $L_{\gamma^{*}}\left(\psi_{1}\left(\beta, \Gamma_{n}\right)\right), \gamma^{*}$ chosen as in Theorem 2.4 with $\alpha \equiv$ identity. The lemma follows by definition in light of our remark above concerning $\Gamma_{n}$.

COROllary. Let $\Gamma$ be a lattice in $N_{n}$. Then

$$
\Gamma(m)=\left\{v \in N_{n}: L_{v} \mid H_{m}(\Gamma)=\text { identity }\right\} .
$$

Conversely, $H_{m}(\Gamma)=\left\{F \in \mathcal{L}^{2}\left(\Gamma \backslash N_{n}\right): L_{\gamma} F=F\right.$ whenever $\left.\gamma \in \Gamma(m)\right\}$.

Proof. It is not difficult to see that the smallest $R$-invariant subspace containing $\theta_{1}(\beta, \Gamma)$ is $H_{1}(\Gamma)$. Hence, since $\mathcal{L}$ and $R$ commute, the previous lemma implies $\Gamma=\left\{v \in N_{n}: L_{v} F=F\right.$ whenever $\left.F \in \theta_{1}(\beta, \Gamma)\right\}$. The corollary follows.

Corollary. For two lattices $\Gamma_{1}, \Gamma_{2}$ in $N_{n}$, we have $\Gamma_{1}(m)=\Gamma_{2}(m)$ if and only if $H_{m}\left(\Gamma_{1}\right)=H_{m}\left(\Gamma_{2}\right)$.

3. Classical theta function theory. Let $D$ be a lattice in $\mathbf{C}^{n}$. The central problem is the question of the existence of nontrivial meromorphic functions on the complex torus $D \backslash C^{n}$. As is well known, this problem is equivalent to the question of the existence of a nontrivial theta function (defined below) on $D \backslash C^{n}$. In this section we will indicate how the classical theory can be developed in the framework of the Heisenberg group. We begin by recalling some of the essential features of the classical theory. For a complete treatment see Lang [4].

For a lattice $D \subset C^{n}$, by a Riemann form on $C^{n}$ with respect to $D$ we mean an $R$-bilinear form $\tilde{A}: \mathrm{C}^{n} \times \mathrm{C}^{n} \rightarrow R$ which satisfies the following properties.

(1)(a) The form $\tilde{A}$ is alternating.

(b) It takes integral values on $D \times D$.

(c) The form $(x, y) \rightarrow \tilde{A}(i x, y)$ is symmetric and positive semidefinite.

We shall work solely with nondegenerate Riemann forms which have the added condition that the form in (c) is both symmetric and positive definite. Not every lattice $D \subset C^{n}, n>1$, admits a nondegenerate Riemann form. For those lattices $D$ which do, we call the corresponding complex torus $D \backslash C^{n}$ an abelian manifold. For the purpose of this work we shall assume that if $D \backslash C^{n}$ has $n$ algebraically independent meromorphic functions defined on it, then $D$ admits a nondegenerate Riemann form. The question we pose is the converse: Show that if $D$ admits a nondegenerate Riemann form, then $D \backslash C^{n}$ has defined on it $n$ algebraically independent meromorphic functions. The proof 
we give is essentially that of C. L. Siegel except in a new framework. We will also investigate the structure of these function spaces.

Recalling the identification $C^{n} \cong R^{2 n}$ above we can associate to the form $\tilde{A}$ a $2 n \times 2 n R$-matrix, also denoted by $A$, uniquely determined by the condition $\tilde{A}(\xi, \eta)=\left(\eta_{1}, \eta_{2}\right)^{t} A\left(\xi_{1}, \xi_{2}\right)$, where $\xi=\xi_{1}+i \xi_{2}, \eta=\eta_{1}+i \eta_{2}$. We will now describe those lattices $D$ for which $D \backslash C^{n}$ is an abelian manifold.

The condition for $A$ to be a nondegenerate Riemann form with respect to a lattice $D$ is given in matrix language by

(2)(a) $A^{t}=-A$.

(b) $\eta^{\prime} A \xi \in \mathrm{Z}$ whenever $\xi, \eta \in D$.

(c) $A J_{2 n}^{t}$ is symmetric and positive definite.

Lemma 3.1. Let $A$ be a $2 n \times 2 n R$-matrix satisfying (a) and (c) of (2). Then there exists an $R$-matrix $C$ satisfying $A=C^{t} J_{2 n} C=C^{t} C J_{2 n}$.

Proof. Since $A^{\prime}=-A$ and $A J_{2 n}^{t}$ is symmetric, $A$ has the property that $A J_{2 n}=J_{2 n} A$. Since, moreover, $J_{2 n}^{t} A=A J_{2 n}^{t}$ is positive definite, it follows that $Y+i X=\bar{C}_{1}^{\prime} C_{1}$, where $A=\left({ }_{-Y}^{X} Y\right.$ X $)$ and $C_{1}=C_{1}^{\prime}+i C_{1}^{\prime \prime}$ (see Lang [4, p. 374]). Put

$$
C=\left(\begin{array}{cc}
C_{1}^{\prime} & -C_{1}^{\prime \prime} \\
C_{1}^{\prime \prime} & C_{1}^{\prime}
\end{array}\right)
$$

and the lemma follows.

The condition on the $2 n \times 2 n$ real matrix $C, J_{2 n} C=C J_{2 n}$, implies that $C$ induces a $\mathrm{C}$-linear mapping on $\mathrm{C}^{n}$. It follows that if we are given a lattice $D$ in $\mathrm{C}^{n}$ admitting a Riemann form $A$, then a C-linear mapping $C$ of $\mathrm{C}^{n}$ exists such that $C(D)$ is a lattice in $C^{n}$ and $J_{2 n}=\left(C^{-1}\right)^{t} A C^{-1}$ is the corresponding Riemann form. In particular, we can restrict our attention to the Heisenberg lattices $D$ of $\mathrm{C}^{n}$ considered in $\S 1$. We can summarize this discussion as follows.

LEMma 3.2. Let $D$ be a lattice admitting a Riemann form $A$. Then there is a C-linear mapping $C$ of $\mathrm{C}^{n}$ having determinant 1 such that $C(D) \in h L\left(C^{n}\right)$ and $J_{2 n}=\left(C^{-1}\right)^{t} A C^{-1}$.

Eventually, we will relate function theory of $\Gamma \backslash N_{n}$ to that of $D \backslash \mathrm{C}^{n}$ where $\pi \Gamma=D$. Essentially, $\Gamma$-periodic functions on the 'nonabelian' object $\Gamma \backslash N_{n}$ are transformed into functions on $\mathrm{C}^{n}$ which, although not $D$-periodic, satisfy a functional equation with respect to the action of $D$.

Recall the short exact sequence $1 \rightarrow Z \rightarrow N_{n} \stackrel{\pi}{\rightarrow} C^{n} \rightarrow 1$ where $\pi(\xi, z)=\xi$. For purposes of the classical theory, it is most natural to consider the spaces

$$
\theta_{m}(\Gamma)=\theta_{m}\left(J_{2 n}, \Gamma\right), \quad m \in Z_{+}^{x} .
$$

We begin by considering results which do not depend on a lattice. Put 


$$
\theta_{d}=\theta\left(J_{2 n}\right) \cap C_{d}^{\infty}\left(N_{n}\right)
$$

where $C_{d}^{\infty}\left(N_{n}\right)$ consists of all $F \in C^{\infty}\left(N_{n}\right)$ of the form $F(\xi, z)=e^{2 \pi i d z} F_{1}(\xi)$, $d>0$ and real. Set

$$
M_{d}=M_{d}(\xi, z)=e^{-2 \pi i d z} e^{-\pi i d x \cdot y} e^{\pi d y \cdot y}, \quad \xi=(x, y) \in R^{2 n} .
$$

Lemma 3.3. The mapping $F(\xi, z) \rightarrow M_{d}(\xi, z) F(\xi, z)$ defines an isomorphism from $\theta_{d}$ onto $E\left(C^{n}\right)$, the entire function on $\mathrm{C}^{n}$.

Proof. Let $F(\xi, z)=e^{2 \text { nidz }} F_{1}(\xi) \in \theta_{d}$. Since $(\mathbf{X}+i \mathbf{Y}) F=0$, we conclude $F_{1}$ satisfies $\pi d(x+i y) F_{1}+(\partial / \partial x+i \partial / \partial y) F_{1}=0$. This latter result implies that

$$
M_{d}(\xi, z) F(\xi, z)=e^{\pi d y \cdot y} e^{-\pi i d x \cdot y} F_{1}(\xi)
$$

is a solution of the Cauchy-Riemann equations. The converse follows by reversing the steps.

CoRollaRy. $M_{m}(\xi, z) \theta_{m}(\Gamma) \subset E\left(C^{n}\right)$ for all lattices $\Gamma$ in $N_{n}$.

For $F \in \theta_{m}(\Gamma), M_{m}(\xi, z) F(\xi, z)$ is entire. We will now translate the $\Gamma$ periodicity of $F$ to the entire function $M_{m}(\xi, z) F(\xi, z)$. To each lattice $\Gamma$ of $N_{n}, \pi(\Gamma)$ is a Heisenberg lattice in $\mathrm{C}^{n}$. Set $D=\pi(\Gamma)$. There are many lattices in $N_{n}$ which determine $D$ in this way. These problems have already been accounted for in the classical theory. We will see how they translate into our language.

Let $D$ be a Heisenberg lattice and set $d=\beta(D)$. Recall $J_{2 n}(D \times D)=d$. $Z, d>0$. Let $K: \mathrm{C}^{n} \rightarrow R$ be an $R$-linear mapping and $B: C^{n} \times C^{n} \rightarrow R$ an $R$-bilinear form satisfying

(6)(a) $B-B^{t}=J_{2 n}$.

(b) $B(D \times D) \subset d Z$.

Corresponding to the pair $K$ and $B$ we shall describe a lattice $\Gamma$ in $N_{n}$ satisfying $\pi \Gamma=D$. Firstly, condition (a) implies $B=G+\frac{1}{2} J_{2 n}$ where $G$ is a symmetric $R$-bilinear form. Define the section $\rho: \mathrm{C}^{n} \rightarrow N_{n}$ to $\pi$ by the formula

$$
\rho(\xi)=\left(\xi, K(\xi)+\frac{1}{2} B(\xi, \xi)\right), \quad \xi \in C^{n} .
$$

LemmA 3.4. Let $K: C^{n} \rightarrow R$ be an R-linear map and $B: C^{n} \times C^{n} \rightarrow R$ an $R$-bilinear form satisfying (6). Let $\rho$ be the associated section. Then $\Gamma=$ $\mathrm{gp}(\rho(D))$ is a lattice in $N_{n}$ and $\pi \Gamma=D$.

Proof. Without loss of generality, we can assume $D=D(l), l=(1$, $\left.l_{2}, \ldots, l_{n}\right) \in Z_{n}^{\#}$. Put

$$
\Gamma^{\prime}=\operatorname{gp}\left(\rho\left(e_{j}\right), \rho\left(l_{j} e_{j+n}\right)\right)_{1<j<n^{\circ}}
$$

We must show $\rho(D) \subset \Gamma^{\prime}$. Take $\gamma \in \rho(D)$ and write 


$$
\gamma=\left(\xi, K(\xi)+\frac{1}{2} G(\xi, \xi)\right), \quad \xi \in C^{n} .
$$

Write $\xi=(x, y)$ and put

$$
\gamma^{\prime}=\left(\xi, K(\xi)+\frac{1}{2}\left(G(x, x)+G(y, y)+J_{2 n}(x, y)\right)\right)
$$

which clearly lies in $\Gamma^{\prime}$. Since $\gamma^{-1} \gamma^{\prime}=\left(0, G(x, y)-\frac{1}{2} J_{2 n}(x, y)\right)$ by (b), $\gamma^{-1} \gamma^{\prime} \in\left[\Gamma^{\prime}, \Gamma^{\prime}\right] \subset \Gamma^{\prime}$ and we are done.

Define for a lattice $\Gamma$ in $N_{n}$,

$$
C_{m}^{(r)}(\Gamma)=C^{(r)}\left(N_{n}\right) \cap H_{m}(\Gamma) .
$$

Lemma 3.5. Let $D \in H L\left(C^{n}\right)$ and choose $K$ and $B$ and $\Gamma$ as in the previous lemma. Then $M_{m}(\xi, z) C_{m}^{(r)}(\Gamma)$ consists of the space of all $C^{(r)}$-functions $\theta$ on $C^{n}$ which satisfy the following functional equation:

$$
\theta(\xi+\eta)=\theta(\xi) e^{-2 \pi i m(L(\xi, \eta)+K(\eta)+B(\eta, \eta) / 2)} e^{-\pi i m L(\eta, \eta)},
$$

where $\xi=x+i y \in \mathrm{C}^{n}, \eta=r+i s \in D, L(\xi, \eta)=s \cdot \xi$.

PRoof. Let $F \in C_{m}^{(r)}(\Gamma)$ and write

$$
\theta(\xi)=e^{-\pi i m x \cdot y} e^{\pi m y \cdot y} F(\xi, 0) .
$$

Then

$$
\theta(\xi+\eta)=e^{-\pi i m(x+r)(y+s)} e^{\pi m(y+s)(y+s)} F(\xi+\eta, 0) .
$$

However, $(\eta, K(\eta))+\frac{1}{2}(B(\eta, \eta)) \in \Gamma$ and, using the $\Gamma$-periodicity of $F$, we get

$$
F\left(\eta+\xi, K(\eta)+\frac{1}{2} B(\eta, \eta)+\frac{1}{2} J(\eta, \xi)\right)=F(\xi, 0)
$$

Hence,

$$
\theta(\xi+\eta)=\theta(\xi) \cdot e^{-\pi i m(\eta y+s x+r s)} e^{\pi m(2 s y+s)} e^{-2 \pi i m(K(\eta)+B(\eta, \eta) / 2+J(\eta, \xi) / 2)},
$$

which can be simplified to give the lemma.

Define for $K, B$ satisfying (6) with respect to the lattice $D$,

$$
\mathrm{Th}^{(m)}(K, B ; D)=\text { the space of all entire functions } \theta
$$

$$
\text { on } C^{n} \text { satisfying (9). }
$$

Lemmas 3.3 and 3.5 immediately imply the following.

THEOREM 3.6. Let $D \in H \mathcal{L}\left(C^{n}\right)$ and choose $K, B$ to satisfy (6) with respect to $D$. Let $\Gamma$ be the corresponding lattice with respect to $K, B$ and $D$. Then

$$
M_{m}(\xi, z) \theta_{m}(\Gamma)=\operatorname{Th}^{(m)}(K, B ; D) .
$$

Corollary. $\operatorname{dim}_{\mathrm{C}} \operatorname{Th}^{(m)}(K, B ; D)=|m|^{n} \cdot \mathcal{P}(D)^{1 / 2}$.

We close this section with a discussion of how our methods have determined all classes of theta functions usually considered, as, for example, 
in Lang [5]. There the following definition is made.

Let $D_{1}$ be a lattice admitting a nondegenerate Riemann form $A$. Choose:

(12)(a) An $R$-bilinear form $B_{1}: C^{n} \times C^{n} \rightarrow R$ satisfying $B_{1}\left(D_{1} \times D_{1}\right) \subset Z$ and $A=B_{1}-B_{1}$.

(b) An $R$-bilinear form $L_{1}: \mathrm{C}^{n} \times \mathrm{C}^{n} \rightarrow \mathrm{C}$ which is C-linear in its first variable and $A=L_{1}-L_{1}^{t}$.

(c) An $R$-linear map $K_{1}: \mathrm{C}^{n} \rightarrow R$.

We denote by $\operatorname{Th}\left(L_{1}, K_{1}, B_{1} ; D_{1}\right)$ the space of all entire functions $\theta(\xi)$ on $\mathrm{C}^{n}$ satisfying the following functional equation.

$$
\begin{array}{r}
\theta(\xi+\eta)=\theta(\xi) \exp \left\{-2 \pi i\left(L_{1}(\xi, \eta)+\frac{1}{2} L_{1}(\eta, \eta)+K_{1}(\eta)+\frac{1}{2} B(\eta, \eta)\right)\right\} \\
\xi \in C^{n}, \quad \eta \in D .
\end{array}
$$

By Lemma 3.2 there exists a complex linear isomorphism $C$ of $C^{n}$ such that the mapping $\theta \mapsto \theta \cdot C$ defines an isomorphism from $\operatorname{Th}\left(L^{\prime}, K, B ; D\right)$ onto $\operatorname{Th}\left(L_{1}, K_{1}, B_{1} ; D_{1}\right)$, where $L^{\prime}=C^{t} L_{1} C, K=C^{t} K_{1} C, B=C^{t} B_{1} C$ and $D=$ $C^{-1}\left(D_{1}\right)$ is a lattice in $C^{n}$ having $J_{2 n}$ as its corresponding Riemann form. Let $L(\xi, \eta)=s \cdot(x+i y), \xi=x+i y, \eta=r+i s$. Then since $L^{\prime}-L^{\prime \prime}=L^{\prime \prime}-$ $L^{t}$ we have $L=L^{\prime \prime}+L^{\prime}$, where $L^{\prime \prime}: \mathrm{C}^{n} \times \mathrm{C}^{n} \rightarrow \mathrm{C}$ is a symmetric C-bilinear form. Hence $e^{\pi i L^{\prime \prime}(\xi, \xi)}$ is entire. Also

$$
e^{\pi i L^{\prime \prime}(\xi, \xi)} \operatorname{Th}\left(L^{\prime}, K, B ; D\right)=\operatorname{Th}(L, K, B ; D) .
$$

The next theorem summarizes this discussion.

THEOREM 3.7. Let $D_{1}$ be a lattice admitting a nondegenerate Riemann form $A$. Choose $B_{1}, L_{1}, K_{1}$ as in (12). Then there exists $a \mathrm{C}$-linear map $C$ of $\mathrm{C}^{n}$ and $a$ symmetric $C$-bilinear form $L^{\prime \prime}$ such that the mapping $\theta \rightarrow e^{\pi i L^{\prime \prime}(\xi, \xi)} \theta(C \xi)$ defines a C-isomorphism between $\mathrm{Th}\left(L_{1}, K_{1}, B_{1} ; D_{1}\right)$ and $\mathrm{Th}^{(1)}(K, B ; D)$, where $D=C^{-1}\left(D_{1}\right)$ is a Heisenberg lattice in $C^{n}$ and $K, B$ satisfy (6) with respect to $D$.

Suppose now that $D$ is a fixed lattice admitting a Riemann form. To each Riemann form $A_{1}$ on $D$ and choice of $L_{1}, K_{1}, B_{1}$ satisfying condition (12) with respect to $D$ and $A_{1}$ we can form the space $Q\left(\operatorname{Th}\left(L_{1}, K_{1}, B_{1} ; D\right)\right)$ of quotients of elements from $\operatorname{Th}\left(L_{1}, K_{1}, B_{1} ; D\right)$. Define

$$
A\left(D \backslash C^{n}\right)=\sum Q\left(\operatorname{Th}\left(L_{1}, K_{1}, B_{1} ; D\right)\right)
$$

where we sum over all $A_{1}, L_{1}, K_{1}, B_{1}$ as above. We call $A\left(D \backslash C^{n}\right)$ the space of abelian functions on $D \backslash C^{n}$.

THEOREM 3.8. Let $D$ be a lattice admitting a nondegenerate Riemann form and $\Gamma$ a lattice satisfying $\pi \Gamma=D$. Then

$$
A\left(D \backslash C^{n}\right)=\sum_{j>0} Q\left(\theta_{j}(\Gamma)\right)
$$


Proof. Fix $K, B$ satisfying (6) with respect to $D$. For any nondegenerate Riemann form $A_{1}$ on $D$ and $L_{1}, K_{1}, B_{1}$ satisfying conditions (12) relative to $D, A_{1}$, there is an integer $m>0$ such that $L_{2}=m L-L_{1}$ is an $R$-bilinear form, C-linear in its first variable and $A_{2}=L_{2}=L_{2}^{+}=m J_{2 n}-A_{1}$ is a Riemann form relative to $D$. Clearly we can form $\operatorname{Th}\left(m L-L_{1}, m K-K_{1}\right.$, $\left.m B-B_{1} ; D\right)$ and choose a nonzero $\alpha$ in this space. For any $f, g \in$ $\operatorname{Th}\left(L_{1}, K_{1}, B_{1} ; D\right)$ both $\alpha f, \alpha g \in \mathrm{Th}(m L, m K, m B ; D)$. Let $\Gamma$ be the lattice determined by $K, B$ as in Theorem 3.6. Then $\operatorname{Th}(m L, m K, m B ; D)=$ $\operatorname{Th}^{(m)}(K, B ; D)$ which equals $M m(\xi, z) \theta_{m}(\Gamma)$.

\section{BIBLIOGRAPHY}

1. L. Auslander and J. Brezin, Translation-invariant subspaces in $L^{2}$ of a compact nilmanifold. I, Invent. Math. 20 (1973), 1-14.

2. J. Igusa, Theta functions, Springer, New York, 1972.

3. N. Jacobson, Lie algebras, Interscience Tracts in Pure and Appl. Math., no. 10, Interscience, New York, 1962.

4. S. Lang, Algebra, Addison-Wesley, New York, 1965.

5. Introduction to algebraic and abelian functions, Addison-Wesley, New York, 1972.

6. A. T. Malcev, On a class of homogeneous spaces, Izv. Akad. Nauk SSSR Ser. Mat. 13 (1949), 9-32.

7. O. T. O'Meara, Introduction to quadratic forms, Springer, Berlin, 1963.

8. C. L. Siegel, Quadratic forms, Lectures on Math., no. 7, Tata Institute of Fundamental Research, Bombay, 1967.

Department of Mathematics, University of Connecticut, Storrs, Connecticut 06268 